\title{
Mechanisms of Contour Perception in Monkey Visual Cortex. I. Lines of Pattern Discontinuity
}

\author{
Rüdiger von der Heydt and Esther Peterhans \\ Department of Neurology, University Hospital Zurich, 8091 Zurich, Switzerland
}

\begin{abstract}
We have studied the mechanism of contour perception by recording from neurons in the visual cortex of alert rhesus monkeys. In order to assess the relationship between neural signals and perception, we compared the responses to edges and lines with the responses to patterns in which human observers perceive a contour where no line or edge is given (anomalous contour), such as the border between gratings of thin lines offset by half a cycle. With only one exception out of 60 , orientation-selective neurons in area V1 did not signal the anomalous contour. Many neurons failed to respond to this stimulus at all, others responded according to the orientation of the grating lines. In area V2, 45 of 103 neurons $(44 \%)$ signaled the orientation of the anomalous contour. Sixteen did so without signaling the orientation of the inducing lines. Some responded better to anomalous contours than to the optimum bars or edges. Preferred orientations and widths of tuning for anomalous contour and bar or edge were found to be highly correlated, but not identical, in each neuron. Similar to perception, the neuronal responses depended on a minimum number of lines inducing the contour, but not so much on line spacing, and tended to be weaker when the lines were oblique rather than orthogonal to the border. With oblique lines, the orientations signaled were biased towards the orientation orthogonal to the lines, as in the Zöllner illusion. We conclude that contours may be defined first at the level of $\mathbf{V} 2$. While the unresponsiveness of neurons in $\mathbf{V} 1$ to this type of anomalous contour is in agreement with linear filter predictions, the responses of $V 2$ neurons need to be explained. We assume that they sum the signals of 2 parallel paths, one that defines edges and lines and another that defines anomalous contours by pooling signals from end-stopped receptive fields oriented mainly orthogonal to the contour.
\end{abstract}

The interpretation of 2-dimensional images in terms of a 3-dimensional world is a basic task of vision. The human visual system performs this task with great ease, so that we hardly become aware of it. Indeed, we see the world 3-dimensionally and if we did not know about the eye's optics and retinal images,

\footnotetext{
Received Sept. 29, 1988; accepted Oct. 24, 1988.

We wish to thank Vappu Furrer-Isoviita and Bernadette Disler for technical assistance and Elisabeth R. Strickler for histological work. Gian F. Poggio helped us to begin the experiments with behaving monkeys, and Günter Baumgartner gave the impulse for this study. The manuscript benefited from comments from Walther H. Ehrenstein, Stephen Grossberg, and two anonymous referees. This work was supported by the Swiss National Foundation Grant 3.939.84.

Correspondence should be addressed to Dr. R. von der Heydt, Neurologische Universitätsklinik, Frauenklinikstrasse 26, CH-8091 Zurich, Switzerland.

Copyright (C) 1989 Society for Neuroscience $0270-6474 / 89 / 051731-18 \$ 02.00 / 0$
}

we would perhaps never suspect that our vision is based on flat images. However, the task involves many problems. Not only is the dimension of depth missing in the images, but information is lacking where part of the scene is hidden from view, and foreground and background are cluttered, i.e., object structures are presented as contiguous that may be separated by a large distance in real space. Thus, apart from the problem of recovering depth, a vision system must be able to separate structures of a partly occluded object from those of the occluding object. It should assign the occluding contour to the forcground object and take into account the incompleteness of the background. The contour may then serve the recognition of the occluding object. Thus, the detection of occluding contours is of primary importance.

In images of 3-dimensional scenes the occluding contours are theoretically defined as the lines of discontinuity of depth since they are borders between the projections of nearer and more distant objects (cf. Marr, 1977; Koenderink, 1984). The problem is to detect these lines in an image. Often they will be marked by a sharp gradient, e.g., a light-dark edge, since foreground and background objects are likely to differ in radiance. However, gradient information is usually not sufficient to delineate objects completely, as the praxis of image analysis by computer has shown. The gradient often vanishes or becomes undetectable at some points, and shadows and surface texture may interfere and lead to false interpretations.

Biological visual systems have developed means to recover the third dimension to some extent, using various cues such as binocular disparity and motion parallax. They cannot, of course, recover information about hidden objects, but they obviously use very efficient methods for recognizing and interpreting situations of occlusion. The cues of binocular disparity and motion parallax are not indispensible for this, at least in humans, who can interpret stationary, flat pictures of 3-dimensional scenes almost as easily as the real scenes. Contours can be perceived even when differences in luminance or chromaticity are absent (Fig. 1). Such contours have been called "apparent edges" (Scheinkanten: Schumann, 1900) or "anomalous contours" (Lawson and Gulick, 1967). Recently, it has been demonstrated that cats can see anomalous contours too (Bravo et al., 1988). This phenomenon shows that perception of contour involves more than just edge detection. ${ }^{1}$

\footnotetext{
Strictly speaking, the contours in figures do not fall under the above definition of contour since they are not lines of discontinuity of depth. Flat pictures can only simulate contours. However, for experimental purposes a 2-dimensional simulation is often preferable to a real 3-dimensional view. The term "anomalous" has the advantage in the present context that it can be used with the above objective definition of contour. We can apply it to physically defined lines, such as the line connecting the tips of lines in Figure $1 C$, whereas terms like "illusory," "subjective," or "cognitive" can only qualify the perception of contour.
} 
Figure 1. Examples of anomalous contours. $A$, The contour of a white oblong; $B$, curved contour; there are no corresponding edges or lines in the figure; $C$ stimulus type used in this study. The anomalous contour was usually moved to and fro across the receptive field, while the circular boundary was kept still. In some experiments, the angle between the anomalous contour and the lines was changed, but the movement was always in the direction of the lines ( $A$ and $B$ reproduced, with permission, from Kanizsa, 1979).
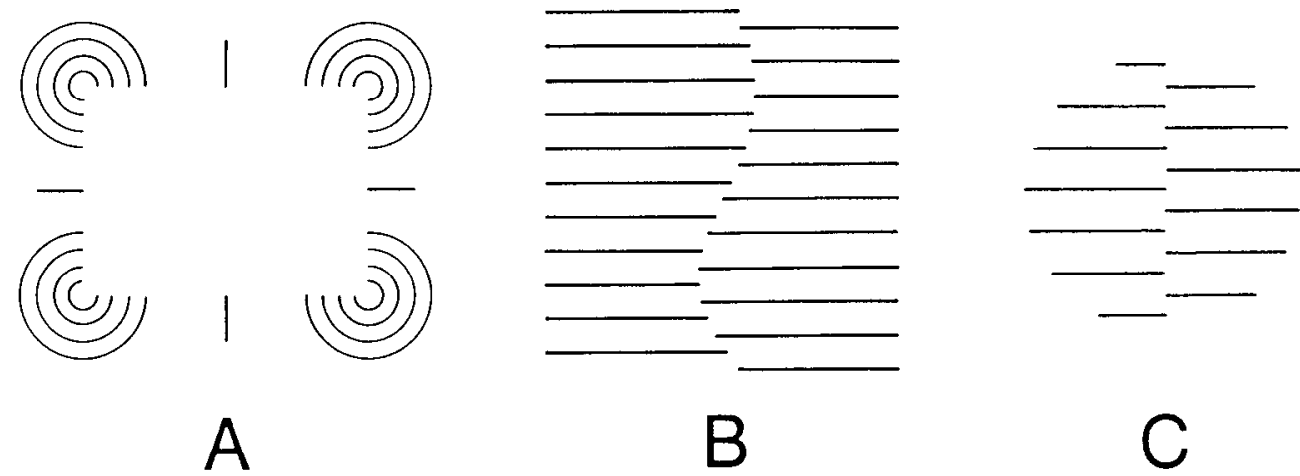

To learn about the neural mechanism of contour perception we recorded the activity of cells in the monkey visual cortex and compared the responses to lines and edges with the activity produced by anomalous-contour figures similar to those of Figure 1. If the activity of orientation-sensitive cortical neurons is related to the perception of contour, as is often assumed, these neurons should respond to edges as well as anomalous-contour figures and signal the orientation of the contour regardless of whether or not it is anomalous. Alternatively, if the responses signal orientation of edges but not anomalous contours, one would have to conclude that these signals represent a stage of processing that is preliminary, or completely unrelated, to the elaboration of contours. Our results indicate that signals in area $\mathrm{V} 1$ of the monkey still represent a preliminary stage, whereas truly contour-related signals, by our definition, are common in area V2.

We report here the results obtained with abutting gratings (Fig. 1C). This stimulus is particularly suitable for our purpose because it consists of lines of only one orientation producing a contour perpendicular to them or at an angle that can be chosen deliberately. In other words, the orientation of the anomalous contour is not shared by any line or edge of the stimulus. Thus, recording from orientation-selcetive ncurons, onc can casily dccide whether a response is related to the anomalous contour or to the elements inducing it. Another important feature of this stimulus is its symmetry about the line of discontinuity (the anomalous contour). The average luminance on lines parallel to the contour is the same on either side. Therefore, the contour does not show up as an edge if the stimulus is blurred or otherwise filtered.

The results obtained with another type of anomalous contour that is akin to the contour of the Kanizsa triangle are treated in a companion paper (Peterhans and von der Heydt, 1989). Some of the results have been reported previously in short form (von der Heydt et al., 1984).

\section{Materials and Methods}

Training. Monkeys (Macaca mulatta) were trained to fixate their gaze on a small target in the center of a stimulus display field at a distance of $40 \mathrm{~cm}$. The fixation target consisted of 2 parallel lines $7 \mathrm{~min}$ arc long and $1 \mathrm{~min}$ arc wide, spaced $5 \mathrm{~min}$ arc from center to center, and the task required a response to a $90^{\circ}$ rotation of the target which could be detected only under foveal fixation. Fixation was checked by watching the eyes at high magnification on a TV monitor.

Upon appearance of the target, the monkey could initiate a trial by pulling a lever. After a variable delay of $0.5-5 \mathrm{sec}$ the target turned and then disappeared after another $0.4 \mathrm{sec}$. During this interval the monkey had to release the lever in order to get a reward in form of a small amount of water or juice. If he released too early or too late, the sequence was unchanged but no reward was delivered. After a pause of $2 \mathrm{sec}$, the target came on again for a new trial. Only mild deprivation was used; the average fluid intake was $300 \mathrm{ml} / \mathrm{d}$ during the training and recording periods. Following extensive training, each of the 3 monkeys used in this study made over $95 \%$ correct responses in about 3000 trials a day on the average over a total of 100-120 d of recording.

Preparation and recording. The animals were prepared for semichronic recording under general anesthesia and aseptic conditions. Anesthesia was initiated by intramuscular injection of $5-10 \mathrm{mg} / \mathrm{kg}$ ketamine hydrochloride and subcutaneous injection of $0.05-0.1 \mathrm{mg} / \mathrm{kg}$ atropinum sulfuricum, and maintained by intraperitoneal injection of $25 \mathrm{mg} / \mathrm{kg}$ pentobarbital sodium (Nembutal). Antibiotics were used only locally. A stainless steel bolt for suspending the animal's head was attached to the skull. Stainless steel cylinders for recording were mounted over the operculum of either hemisphere in succession. One or $2 \mathrm{~d}$ before a series of recording sessions, a trepanation of $3 \mathrm{~mm}$ diameter inside a cylinder was made under anesthesia. Single units were then recorded by inserting a microelectrode through the dura, once a day, until it became impossible to insert the electrodes undamaged or signs of dimpling were observed. This was usually the case after 10-14 d. After making a new trepanation, another series of recording sessions was begun, and so on. Typically, 5 trepanations were placed in each cylinder.

Microelectrodes were glass-coated platinum-iridium wires prepared according to Wolbarsht et al. (1960) but without platinum-black coating. The wire was $0.1 \mathrm{~mm}$ in diameter, the etched tips had tapers between 0.07 and 0.09 , and the coated electrodes had impedances of $3-5 \mathrm{M} \Omega$ at $1 \mathrm{kHz}$. These electrodes isolated cortical units well and also picked up multiunit activity at audible levels. On average, 22 units could be discriminated in vertical penetrations of the striate cortex. A typical electrode track passed through striate cortex, white matter, and the prestriate cortex in the posterior bank of the lunate sulcus, which was usually V2. Often striate and prestriate cortex were penetrated on subsequent days at the same track position. Advancing the electrode, we carefully monitored the entry into the cortex, the amount of single and multiunit activity and its stimulus preferences such as orientation and ocularity, the entry into the white matter, etc. The corresponding depths were recorded graphically. Comparison of such track charts with the histological reconstructions showed that layers $4 \mathrm{~B}, 4 \mathrm{C}$, and 6 in V1 could often be identified, the entrance into $4 \mathrm{~B}$ by a drop in unresolved activity and a low density of isolatable units, $4 \mathrm{C}$ by its unresolved, monocularly driven, and orientation-nonselective activity (Poggio et al., 1977), and layer 6 again by a higher density of isolatable units.

Histology. Toward the end of recording, several tracks were marked by electrolytic lesions. To mark the area of recording, $4-8$ sharply pointed tungsten pins $0.25 \mathrm{~mm}$ in diameter were inserted, under anesthesia, using the electrode positioning device. The animal was then deeply anesthetized and the brain perfused through the heart with Ringer solution containing $5 \mathrm{U}-\mathrm{USP} / \mathrm{ml}$ heparin followed by $4 \%$ formaldehyde. In 2 monkeys, the marked blocks of tissue were frozen or embedded in celloidin and cut; in the third, they were cut on the vibratome. The slices were stained with cresyl violet or thionine. The shrinkage factor was determined from the distance of the marker pins, and the electrode tracks were drawn in on enlarged photographs or drawings of the sections. The electrolytic lesions were found within $200 \mu \mathrm{m}$ of the reconstructed tracks.

Visual stimulation and response analysis. The visual stimuli werc generated by means of analog and digital circuits on an $x-y$ oscilloscope (R. von der Heydt and V. Corti, unpublished observations). The oscil- 
loscope was a flat-faced, flying-spot scanner cathode-ray tube with magnetic deflection and focus (Ferranti $7 / 21$ ) equipped with a very fast decaying, yellowish green phosphor (Ferranti A5, peak at $555 \mathrm{~nm}$ ). Basically, 2 linear ramp signals generated a raster of 240 lines that could be electronically rotated and positioned on the screen. The various shapes of stimuli such as light and dark bars, gratings, or the anomalouscontour figure of Figure $1 C$ were formed by modulating the intensity. The raster was written alternatingly with the fixation target within 5 msec, and both were written alternatingly at 2 positions of the screen and thence projected separately in the 2 eyes via a stereoscope ( $R$. von der Heydt, unpublished observations). The frame rate in each eye was thus $100 \mathrm{~Hz}$. The 2 rasters could also be positioned or distorted differentially in the 2 eyes for stereoscopic depth effects. The raster size was adjusted for each neuron to match its resolution and size requirements; a $4^{\circ} \times 4^{\circ}$ square was often used, resulting in $1 \mathrm{~min}$ arc line spacing, while higher resolution or greater size was chosen for some neurons. In any case, we could stimulate a stereoscopic field of $21^{\circ}$ diameter at the monkeys eyes, while the spot size of the oscilloscope allowed us to produce lines less than $1 \mathrm{~min}$ arc wide. Stimuli and fixation target appeared superimposed on a $42^{\circ} \times 30^{\circ}$ homogeneous background field (tungsten filament light). The luminance of the stimulus increment was usually $10 \mathrm{~cd} / \mathrm{m}^{2}$, which was also the luminance of the background. Only binary patterns were used in this study. The stimuli were usually oscillated back and forth at constant speed and frequency, e.g., $1 \mathrm{~Hz}$. The anomalous-contour stimulus was bounded by a stationary fieldstop, not visible by itself, so that only the contour was seen in movement. In early experiments, a physical, circular field-stop of $6^{\circ}$ was used and, later, an electronical, square window of similar size. For control by the experimenter, the stimuli were also displayed on a slave scope. Here, the stimuli of both eyes were superimposed, and coincided when disparity was zero. The monkey's fixation periods were signaled by a brightening on the slave scope. Stimulus parameters such as orientation and length or number of lines and degree of overlap of the gratings could be set manually or varied automatically in pscudorandom order.

The electrode signal was fed into an audio monitor, a window discriminator, and a delay line in parallel. The output pulses of the discriminator triggered a display of the delayed signal that showed the spike form and were fed into a dot display unit and a computer. The dot display showed the activity during stimulus cycles that fell within a fixation period, ordered in groups according to the stimulus parameter. Upon completion of a stimulus sequence, e.g., 8 cycles at each of 16 orientations, it was photographed on instant film. Figures 2, 3, and 19 show examples. The computer counted the numbers of action potentials in each half-cycle and for each stimulus condition and displayed and stored the means and standard deviations. The preferred orientations and widths of orientation tuning were determined from curves through 16 equidistant data points, covering $45^{\circ}, 90^{\circ}$, or $180^{\circ}$, by calculating midpoint and distance betwecn the points of half-maximal response on either flank. Simple cells were distinguished from complex cells by the separation of the traces in the dot display of responses to light and dark bars, and light and dark edges.

We found it important to have easy manual-visual control over the stimulus-using a joystick, a set of calibrated potentiometers, the slave scope, and a digital display of the actual stimulus parameter-and at the same time to be able to use any stimulus setting immediately as the starting point for a series of recordings. The raster display and the graphic representation of the recorded responses then provided a check on the observations made by listening to the responses.

\section{Results}

Sincc orientation is an important perceptual quality of contours, we have concentrated our analysis on the orientation-selective neurons in the visual cortex. Each receptive field was investigated first with edge and bar stimuli. By varying the stimulus and listening to the responses, we determined the best stimulus dimensions, amplitude, and velocity of motion, binocular disparity, and preferred orientation. Using these settings, we mapped the response field, i.e., the minimum region outside of which the stimulus evoked no response. Then, we tested the anomalous-contour stimulus (Fig. $1 C$ ). We moved the line of discontinuity over the response field and tried various orientations and line spacings. Gratings with 24 and $48 \mathrm{~min}$ arc line spacing were used mostly because these seemed to us to give the most vivid and typical perception of anomalous contour in nearfoveal vision, but other spacings, from 6 to $96 \mathrm{~min}$ arc, were also tested if the former gave only weak or no responses. We then determined the orientation tuning with the edge, or bar, and with the anomalous contour. A total of 193 neurons were studied, 60 in V1, 103 in V2, and 30 near the border between these 2 areas $( \pm 0.5 \mathrm{~mm})$. The receptive fields of the cells were all located in the lower hemifield, those of $\mathrm{V} 1$ at radial excentricities between $0.9^{\circ}$ and $4.6^{\circ}$ (mean $2.0^{\circ}$ ), and those of $\mathrm{V} 2$ and the border region between $1.1^{\circ}$ and $6.7^{\circ}$ (mean, $\left.2.8^{\circ}\right)$.

\section{Orientation of the anomalous contour}

Figures 2 and 3 show examples of the responses obtained with the 2 types of stimuli, a light bar and the abutting gratings, at 16 orientations covering $180^{\circ}$. Stimuli for 2 orientations are depicted. The bar was moved sideways, and the anomalous contour was also moved perpendicularly to its orientation, i.e., the tips of the lines were being extended and withdrawn. Figure 2 represents a neuron recorded in layer 2 or 3 of V1. It responded best to a bar oriented $40^{\circ}$, but no response was obtained with the anomalous-contour stimulus at this orientation. The neuron responded when this stimulus was rotated by $90^{\circ}$ (top traces) or $-79^{\circ}$ (bottom traces), i.e., when the lines of the gratings came close to the neuron's preferred orientation. Thus, one could say that this cell signals "what is there" rather than what we see. Although it is commonly accepted that the activity of neurons in V1 closely reflects the visual stimulus, it is nevertheless remarkable that a line that is so vividly perceived has no correspondence in the responses of a line-detecting neuron.

Figure 3 shows the responses of a cell recorded in layer 6 of V2 under similar conditions. It can be seen that this neuron responded to the anomalous-contour stimulus when the contour had the orientation that was optimal for the bar. Although less than to the bar, it fired regularly at every sweep, and the corresponding orientation tuning was very similar. Thus, we can say that this cell signals something that we see although it is not there. The display at the bottom right shows that a grating without the contour, at the orientation that was optimal for the stimulus with contour, did not evoke a response. Thus, what is signaled by the neuron is indeed the line of discontinuity. A further difference between this and the neuron of Figure 2 is that this neuron was not activated when the abutting gratings were presented with the lines at the preferred orientation; it did not signal the orientation of the gratings. This feature will be considered again below.

Figures 4 and 5 show the typical results obtained with the 2 kinds of stimuli plotted as orientation tuning curves. Responses to bars or edges are plotted with continuous lines, those to the abutting gratings with stippled lines. For the latter, orientation refers to the anomalous contour. Figure $4 \mathrm{~A}$ shows the tuning of the neuron of Figure 2, and Figure $4 B$ of another neuron of V1. In each case, the abutting gratings produced no peak where the bar responses had a maximum, i.e., the responses of these cells did not reflect the orientation of the anomalous contour. With only one exception, this result was invariably obtained in V1. Simple, complex, and end-stopped cells did not differ in this respect. Also, 82 of 133 neurons of $\mathrm{V} 2$ and $\mathrm{V} 1 / \mathrm{V} 2$ border region showed this behavior. The one exception in V1 was recorded in the deep layers, at a distance of $1.3 \mathrm{~mm}$ from that border. The negative results do not mean unresponsiveness to just one stimulus; we have always varied the line spacing and the position 

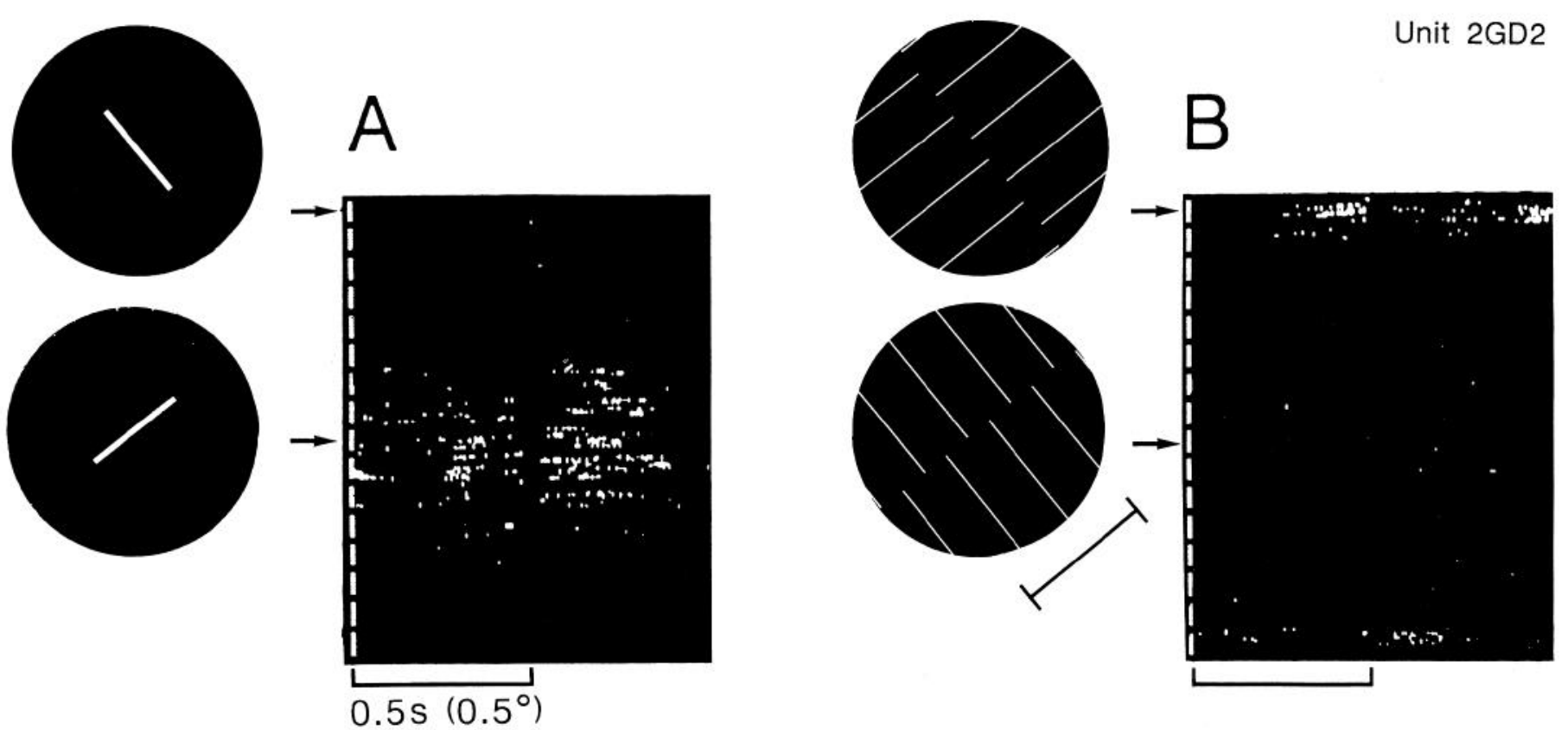

Figure 2. Responses of a neuron recorded in the primary visual cortex (V1) to a light bar $(A)$ and a stimulus with anomalous contour $(B)$. Either stimulus was moved back and forth 8 times at each of 16 orientations covering a range of $180^{\circ}$ ( 2 orientations are illustrated). The spikes are represented by dots. Each horizontal row corresponds to a cycle of movement: the forward sweep is plotted from left to right in the left half, and the backward sweep from right to left in the right half. It can be seen that the cell responded best to a bar oriented at $2-8$ o'clock but did not respond when the anomalous contour was presented at this orientation. An $I$-bar indicates the extent of the receptive field (limit of length summation for the bar, $80 \mathrm{~min}$ arc) for comparison. Supragranular complex cell with light-bar preference. The stimulus insets in this and the following figures are photographs of the actual stimuli, but line thickness has been enhanced and a circular window added. The actual field stop used with the anomalous-contour stimulus was twice as large.

in order to find the optimum. Effective positioning was possible, despite fixational eye movements, since we could drive cells with small receptive fields by thin lines moving lengthwise (cf. responses at the top of Fig. $2 B$ ). In end-stopped cells we have tried also anomalous contours of limited length (e.g., contours produced by 4-6 lines).

Peaks of orientation tuning corresponding to the anomalous contour were found in 45 of 103 cells $(44 \%)$ of V2 (51 of 133 , or $38 \%$, if cells recorded near the V1/V2 border are included). Figure 5 presents examples of such orientation tuning in V2. As demonstrated, the tunings obtained with light bar and anomalous contour were generally quite similar in shape and usually peaked at nearly the same orientation (Fig. 5, $A-C$ ). A few cells clearly preferred slightly different orientations; the case with the largest difference is shown in Figure $5 D$. The strengths of lightbar and anomalous-contour responses were often quite different, and their ratio varied from cell to cell. The neuron of Figure $5 \mathrm{~A}$ responded better to the anomalous-contour stimulus than to bars and edges of any size and contrast, while the neuron of Figure $5 B$ responded much better to a thin light bar. Cells were often studied over several hours, and the responsiveness, or unresponsiveness, to anomalous contours did not change. We did not find a criterion by which one could predict this property from responses to conventional stimuli. The receptive fields of most of these cells appeared to be complex by the criterion that responses to light and dark bars, or to edges of opposite contrast, largely overlapped. Others responded selectively to either dark or light bars, or to edges of one polarity only (cf. Peterhans and von der Heydt, 1989). We found that the position of the grating lines along the anomalous contour (the phase of the gratings) was not critical, and mirror-reversing the stimulus at the contour made no difference.

Responses reflecting the orientation of the gratings. The cells of Figure 5, $A, B$ showed a second peak in response to the grating stimuli, corresponding to the orientation of the lines. This can be seen in $A$; it is not shown in $B$ because we have not recorded the responses at these orientations. The cells of Figure 5, $C, D$ did not show the secondary peak; they seemed to ignore the orientation of the gratings. Another example of this kind is neuron $4 \mathrm{CH} 2$ of Figure 3, whose tuning curve is shown in Figure $11 A$ below. This behavior was found in 23 of 45 cells that signaled the anomalous contour. Unresponsiveness to the gratings was sometimes also observed in V1. As the peaks of stippled curves in Figure 4 show, cell A was excited by the grating nearly to the same degree as by the light bar, but cell B was hardly activated by it at all. We found 2 reasons for this lack of responses. First, the grating stimulus was less effective than a single line of it in the receptive field center. Second, such a line, ending in the receptive field, was still less effective than a line continuing across it; in fact, the response to a long line was more than the sum of the responses to either line end. About a third of the cells lacking anomalous-contour responses did not respond to the abutting gratings at all.

Figure 6 is an attempt to demonstrate the difference between the results obtained in V1 and V2 by the distribution of a single parameter. Our classification was based on the orientation tuning since the question was whether anomalous contours, specifically their orientations, are explicitly represented. However, although the tuning can be easily recognized in the curves, or by varying the orientation and listening to the responses, there 

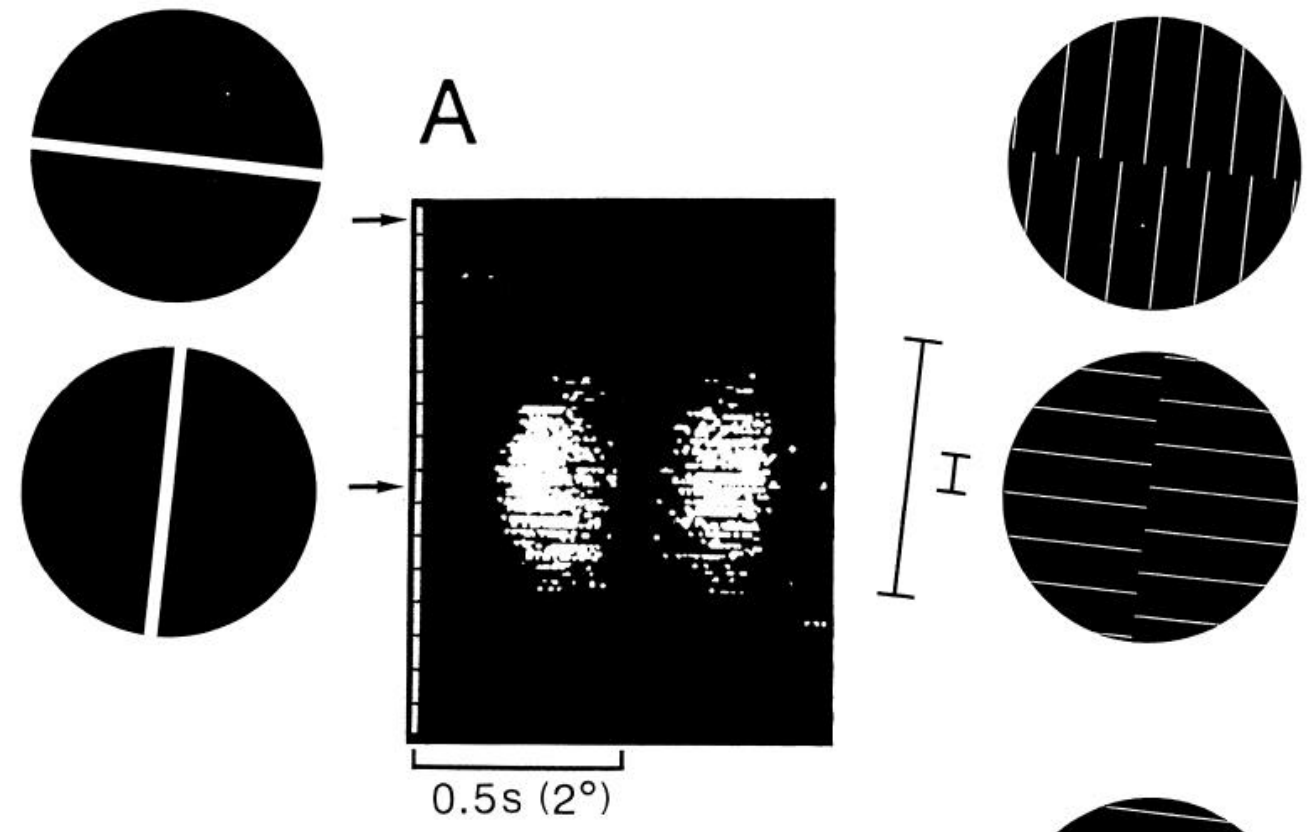

Unit $4 \mathrm{CH} 2$
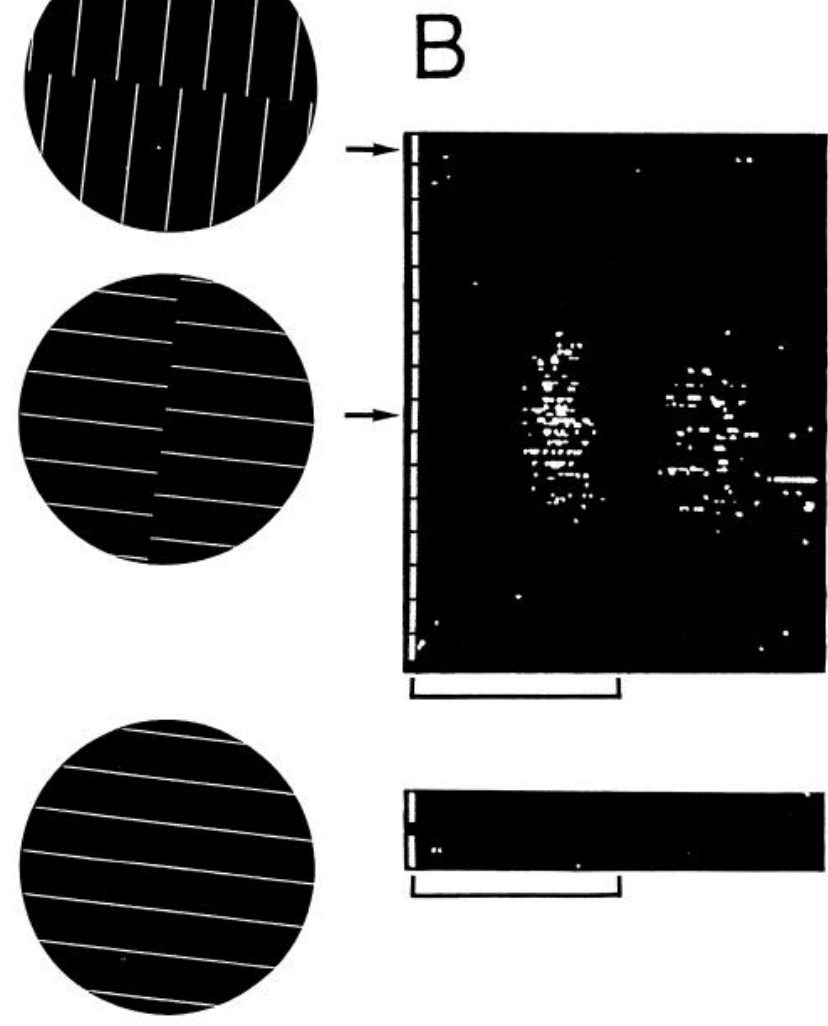

Figure 3. Responses of a neuron of $\mathrm{V} 2$ to light bar $(A)$ and anomalous-contour stimulus $(B)$. Conventions as for Figure 2 . The anomalous-contour stimulus evoked responses just at those orientations at which the bar was effective. The neuron thus signaled orientation of bars as well as anomalous contours. We call such neurons "contour cells." The bottom display shows the result of 16 presentations of a grating without discontinuity that was rocking back and forth between the positions of the abutting gratings used above. It produced no responses. I-bars indicate the size of the response field $(20 \mathrm{~min}$ arc) and the limit of summation for the anomalous-contour stimulus (140 min arc).

is no obvious way to convert it into a single parameter. For simplicity, we have chosen here to calculate just the response ratio: strength of the response to the anomalous-contour stimulus, with the contour at, or close to, the cell's preferred orientation, over strength of the optimum bar or edge response. The number of spikes counted per stimulus cycle minus the count of spontaneous activity was taken as the response strength. One can see that most cells of V1 pile up near zero, while among the cells of V2 there is a group of neurons with relatively large responses to the anomalous contour. The black parts of the histograms represent neurons with a peak in the tuning corresponding to the contour. The other cells with positive response strengths showed a flat tuning or a trough instead. While orientation selective for bars and edges, they resembled unoriented cells in their responses to the anomalous contour. Not included in the histograms are 46 cells that were easily classifiable and whose tunings were not recorded. These are 11 cells of V1 and 26 cells of V 2 with no responses to the anomalous contour and 9 positive cases of $\mathrm{V} 2$.

\section{Orientation tuning and direction selectivity: anomalous contours versus edges and bars}

The finding that many cells in V2 signaled anomalous contours as well as bars and edges was a surprise to us. Another was the observation that the preferred orientations were not always the same for such a contour as for an edge or bar. This is demon- strated in Figure 7, where the distribution of the differences in preferred orientation is plotted for 28 cells of V2. (In 6 other cells for which the tuning curves are also available, we found that a comparison of peaks and widths would not be valid, either because of low signal-to-noise ratio or because one of the curves was strongly asymmetric.) In Figure $7 A$ the differences are given in absolute terms; in Figure $7 B$, relative to the widths of orientation tuning at half-amplitude for the edge or bar. It can be seen that the differences were small $\left(<7.5^{\circ}\right)$ in most cells and were never greater than $35^{\circ}$. In several cells, however, the difference was comparable to the width of tuning.

Also, the widths of orientation tuning were not exactly the same for the 2 kinds of stimuli. The scatter plot of Figure 8 shows the 2 widths for each of the 28 cells. The 2 agreed in the mean $\left(46^{\circ} \pm 18^{\circ}\right.$ for the bar/edge vs $44^{\circ} \pm 15^{\circ}$ for the border between gratings) and were correlated at $R=0.84$, indicating some independence. Also remarkable is the large range of tuning widths, from $12^{\circ}$ to more than $70^{\circ}$, of the responses to either anomalous-contour or conventional stimuli.

We have also looked at the direction sensitivity of these cells for the 2 kinds of stimuli. Figure 9 shows a scatter plot of the indices of directionality [(preferred-nonpreferred)/preferred]. It can be seen that nearly any degree of direction sensitivity was observed among these neurons. In the major group of cells represented in the upper part of the plot, a positive correlation between the 2 indices is obvious. A group of 5 cells showed 


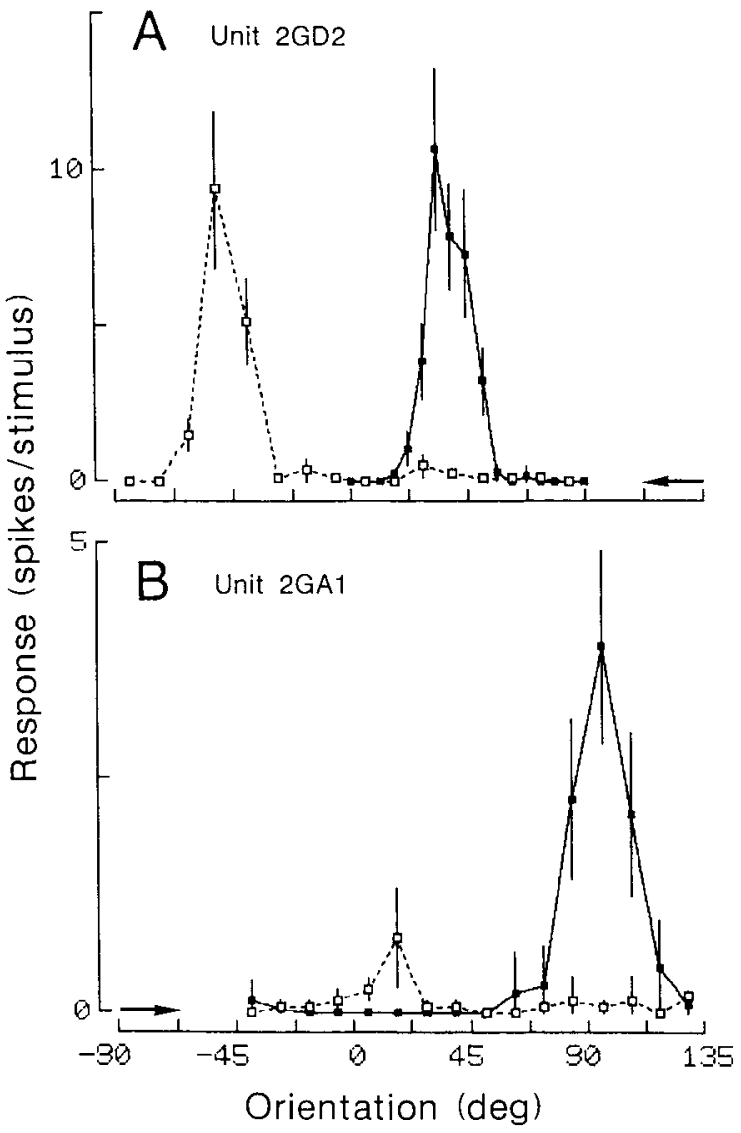

Figure 4. Typical examples of orientation response curves obtained with light bar and abutting gratings in cells of V1. Continuous curves represent the light-bar responses, dotted curves the responses to abutting gratings, plotted with reference to the anomalous contour. Horizontal arrows indicate the levels of spontaneous activity. The cells were sharply tuned to the orientation of bars, but neither showed a response for the anomalous contour at the corresponding orientation. In $A$ (the neuron of Fig. 2), a peak appears at $90^{\circ}$ from the peak of the bar responses; in other words, this neuron signaled the orientation of the gratings. In $B$ the neuron hardly responded at all to the abutting gratings. $A$, Supragranular complex cell; $B$, simple cell from layer $4 \mathrm{~B}$.

opposite (though weak) direction preferences for bar/edge and anomalous-contour stimuli, and we do not know how to interpret this result.

\section{Variations of the stimulus}

The observation that some neurons respond when an anomalous contour is passing over their receptive field and show similar orientation selectivity for this contour as for bars or edges suggests that they signal contours. However, a number of questions are still open. Our stimulus consisted of lines and was moved linearly. Are the neurons perhaps signaling direction of movement rather than orientation, or the orientation perpendicular to the grating? Can the anomalous-contour responses be explained on the basis of linear spatial filter theory? How do they relate to the responses of the well-known cell types, simple and complex, and their end-stopped (hypercomplex) counterparts? Do they depend on the length of contour and number of inducing lines, and can responsiveness to anomalous contours be related to conventional length-summation properties? Alternatively, one might recall that end-stopped receptive fields can be activated by short edges, or tongues (Hubel and Wiesel, 1965, 1968), and the end of a line can be conceived as a very thin (1-min-arc wide) tongue. In this case, a line orthogonal to the cell's preferred orientation would produce a response. The following experiments were carried out to address these questions.

Moving versus stationary. Movement is not a necessary condition for the anomalous-contour responses. In the cell shown in Figure $5 D$, for example, we used stationary stimuli: The pattern of abutting gratings was alternated with a grating without discontinuity, making the anomalous contour appear and disappear, and the bar was flashed on and off. Orientation tuning with stationary anomalous contours was recorded in 3 other cells. Figure 10 shows a comparison between the moving and stationary conditions. In this case, the stationary stimuli were continuously displayed at the various orientations and the activity during periods of fixation was measured in the same way as for the moving condition. The spike counts were higher with stationary presentation for either kind of stimulus, but the tunings were very similar. Thus, movement was not relevant for the orientation tuning; the cell signaled orientation rather than direction of movement.

Axis of motion and angle of inducing lines. Are the responses determined by the orientation of the anomalous contour or do they also depend on the orientation of the inducing lines? We have shown in Figure $3 B$ above that a rocking line grating without discontinuity did not produce a response; however, for the responses evoked by the discontinuity, the orientation of the lines could nevertheless have an influence on the tuning. A similar argument could be made concerning direction of movement. One can separate the effects of both, line orientation, and movement direction from that of the anomalous-contour orientation by varying the angle between the lines and the contour, keeping the direction of movement parallel to the lines. We have recorded orientation tuning curves with patterns of 3 angles $\left(45^{\circ}, 90^{\circ}\right.$, and $\left.135^{\circ}\right)$ in 8 cells. Figure 11 shows 3 cxamples. Each curve plots response against orientation of the border between the gratings. The curves of neuron A all peak at the same orientation, i.e., the different line orientations had no effect. Neuron B also signaled precisely the same orientation for 2 of the patterns but did not respond to the third pattern (except at $-40^{\circ}$, where it signaled the orientation of the lines). In neuron $\mathrm{C}$, the peaks for the oblique-line patterns were shifted to either side of the peak for the $90^{\circ}$ pattern. The directions of the shifts indicate that this neuron sensed orientations lying between the orientation of the border and that orthogonal to the grating lines. In the other cells, the deviations were between those of neurons $A$ and $C$ in size, and always in the same sense as for neuron $C$. Taking as a measure half the difference between the peak orientations of the $135^{\circ}$ pattern and the $45^{\circ}$ pattern, the oblique lines caused deviations between $1.3^{\circ}$ and $23.7^{\circ}$ (mean, $11.2^{\circ}, n$ - 7). The most sharply tuned cells (Fig. 11, $A, B$ ) showed the smallest deviations. If tuning were determined by the orientation of the lines or the direction of motion, the deviations should have been $45^{\circ}$; in fact, they were much closer to zero, the prediction from the anomalous contour. One may conclude that the cells indeed signaled orientation of contour, but some of them with a bias towards the orientation orthogonal to the lines inducing the contour. (We cannot distinguish from this experiment between the biasing effects of line orientation and direction of motion.) Line orientation also affected the amplitudes of response. In 6 of the 8 cells the mean response to the obliqueline patterns was smaller than that to the orthogonal pattern. 


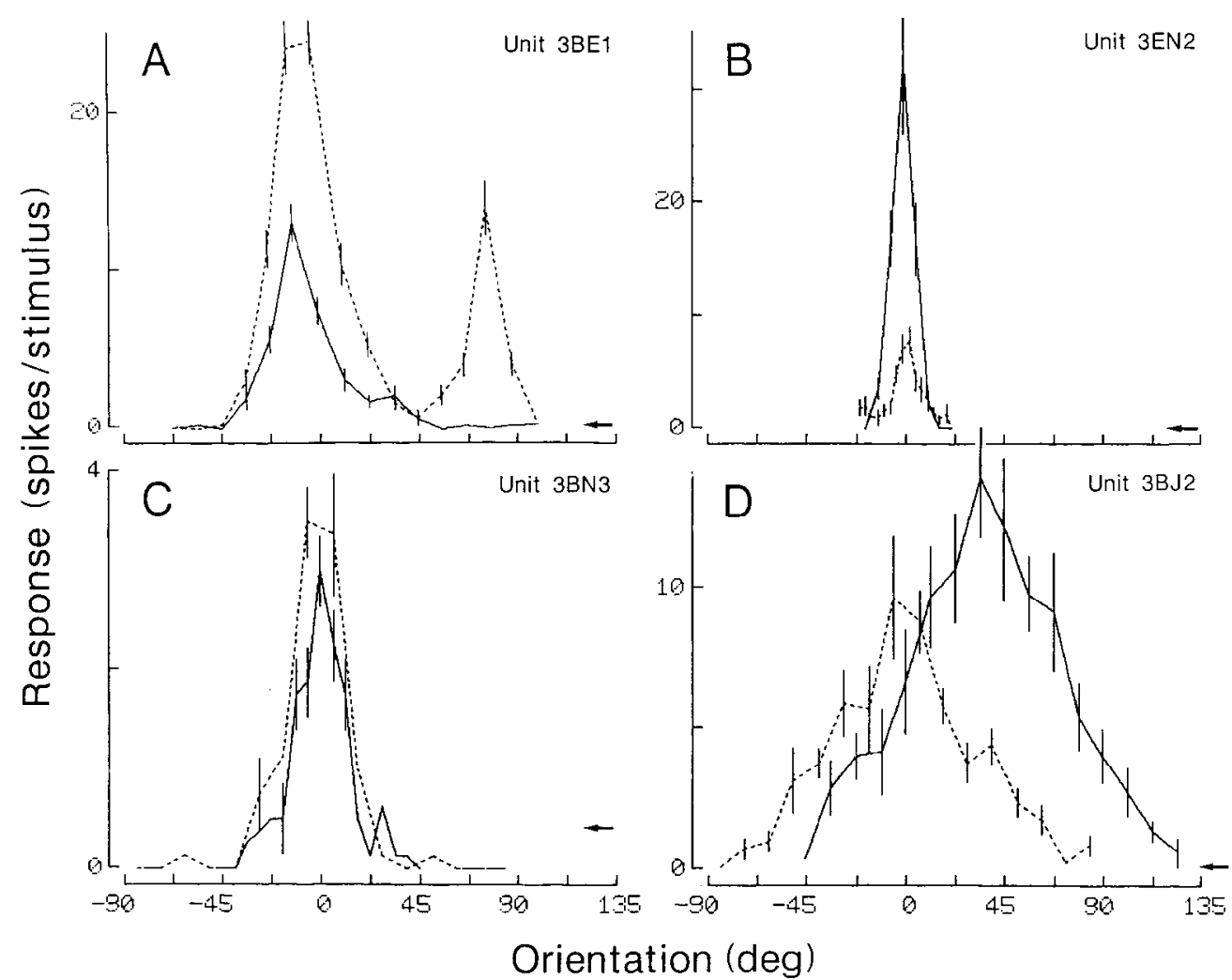

Figure 5. Orientation tunings obtained with light bar and abutting gratings in 4 "contour cells" of V2. Again, continuous lines stand for bars, broken lines for anomalous contours, and horizontal arrows mark the levels of spontaneous activity. One can see the peaks of tuning corresponding to the anomalous contour. The relative strengths of the 2 kinds of responses varied between cells (cf. $A$ and $B$ ). The peaks were usually quite similar for either kind of stimulus $(A-C)$, but in some cells they were displaced $(D)$. The curve for the anomalous-contour stimulus of neuron $A$ has a second peak corresponding to the orientation of the grating lines. Neuron B gave a similar response (not shown). Neurons C and D did not signal the orientation of the gratings. Moving stimuli were used for $A-C$ and stationary for stimuli for $D$ (the bar was turned on and off, the abutting gratings were alternated with a grating without discontinuity).
These findings have perceptual analogs that will be discussed below.

The following observations show that the way the anomalouscontour signals are generated is quite different from the mechanism of edge and bar responses in simple, complex, or endstopped cells.

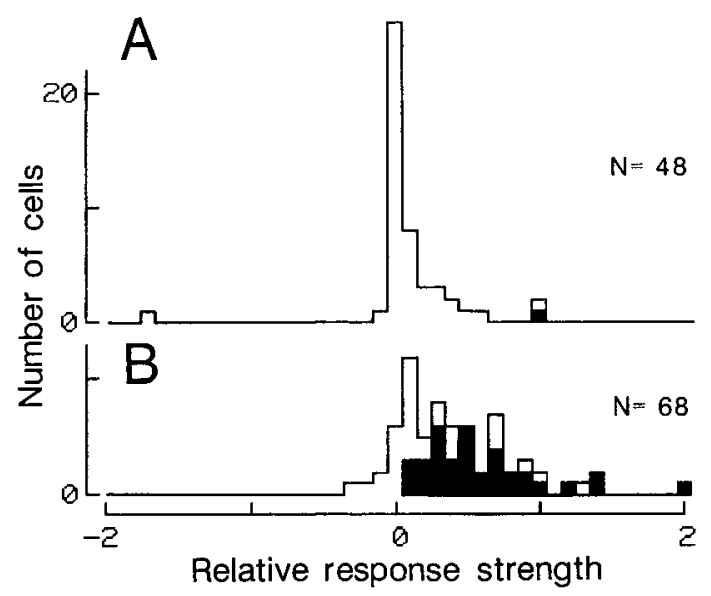

Figure 6. Distributions of the relative strengths of anomalous-contour responses in V1 and V2. For each cell, the response to the abutting gratings, with the contour at the optimum orientation, was divided by the optimum bar or edge response. Negative values indicate suppression of activity below the spontaneous level. $A$, Cells from V1 $(n=48) ; B$, cells from V2 $(n=68)$. The filled areas represent cells that showed a peak in the orientation tuning corresponding to the contour (contour cells); in the other cells with positive response strengths, the tuning was flat or showed a trough instead.

\section{Number and density of lines}

Concerning the idea that ends of lines are equivalent to infinitely short edges or "tongues" that would stimulate end-stopped receptive fields oriented perpendicularly to the l'nes, we found

\section{Difference in optimal orientation}

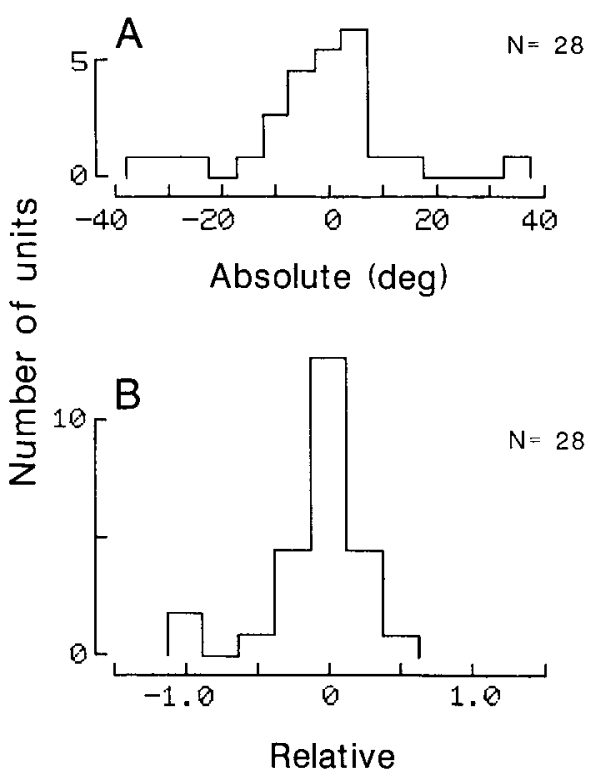

Figure 7. Distribution of the difference between the peak-orientations for bar/edge and anomalous contour for the contour cells of V2. $A$, Absolute orientation differences. $B$, Each difference was first divided by the mean of the widths at half-height of the 2 tuning curves. This shows that the differences were usually much smaller than the widths of tuning. 


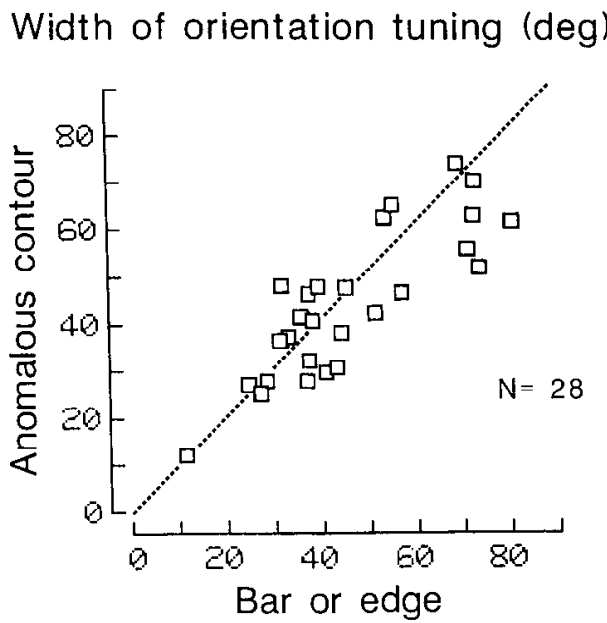

Figure 8. Scatterplot of the widths of the orientation tunings for bars or edges and anomalous contours of V 2 contour cells. Cells with equal widths for both stimuli would fall on the dashed line.

that about half of the cells recorded in V 1 showed end-inhibition ( $50 \%$ reduction or more) and 4 were completely inhibited by long stimuli, but none of these responded to abutting gratings with lines orthogonal to the cell's preferred orientation. Further, for a single line or the end of a single linc, we have never secn a respectable peak of response at this orientation, in neither $\mathrm{V} 1$ nor V2. The contour cells of V2 either did not respond to a single line-end or did not show the orientation selectivity that was observed with the contour (see Fig. 16 below).

The question of whether a single line would suffice is important also from the perceptual point of view. In configurations like those of Figure $1, B, C$, the perception of the anomalous contour depends on the number of the lines that terminate at the contour. A contour is not perceived at the end of just one line; it appears perhaps with 2 or 3 lines, and increases further in "strength" or "distinctness" when more lines are added. We found a similar increase in the strengths of the neuronal responses to these stimuli. Figure 12 shows the result of an ex-

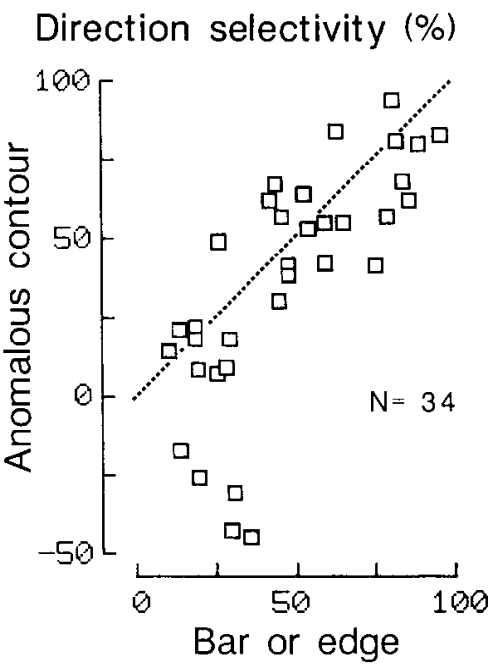

Figure 9. Scatterplot of the indices of dircetion sclcctivity for bars or edges and anomalous contours of V2 contour cells. The dashed line represents equal direction selectivity for both stimuli.

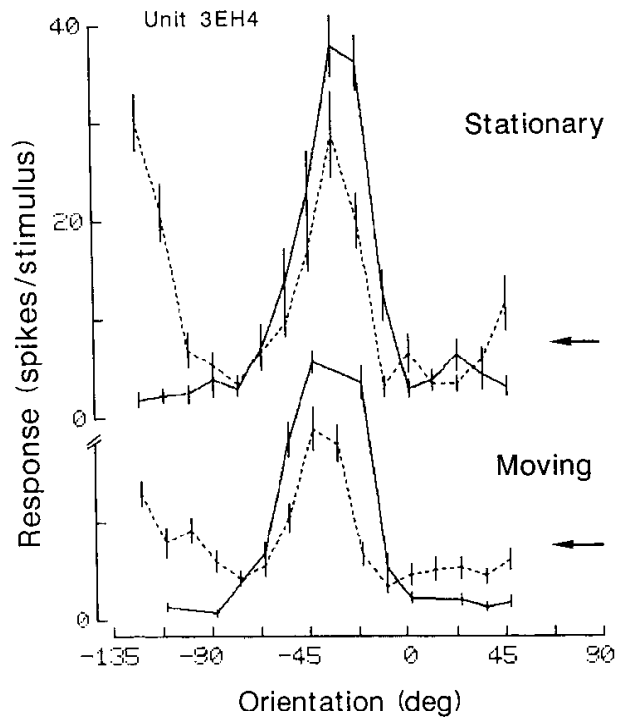

Figure 10. Comparison of moving and stationary stimuli in a contour cell of V2. Orientation tuning. Continuous line, light bar; broken line, anomalous-contour stimulus. Horizontal arrows indicate level of spontaneous activity.

periment in which the pattern of Figure $1 C$ was reduced to various numbers of lines by placing a variable electronic slit symmetrically over the receptive-field center. The 10 graphs show the "summation curves" of 10 cells that signaled the border between gratings. The border was presented at the optimum orientation. It can be seen that the cells usually did not respond to the end of one line moving into and across the receptive field, and responded maximally only to patterns of 8-13 lines. With more lines, the responses either leveled off or decreased again (b). Six of the cells $(a-f)$ exhibited marked thresholds; in the others, the responses increased more or less linearly from zero. The curves of cells $e-g$ and $i$ are reproduced in Figure 17 for comparison with the conventional length-summation curves.

The neurons of Figure 12 differed in the amplitudes of their responses to the anomalous-contour stimulus in absolute terms as well as relative to the maximum edge or bar responses. In order to get some idea of the average strength of the anomalous contour signals, we have normalized the responses of each cell to its maximum bar or edge response and then calculated the mean of the 10 curves. The signals were thus weighted differently, taking the strength of the respective bar or edge response as reference. This mean relative response as a function of the number of lines is shown in Figure 13. It increases linearly and levels off at about 10 lines.

Is it number of lines or length of contour that determines the response amplitudes? In 2 neurons we carried out the above summation experiment with 2 different line spacings, 24 and $48 \mathrm{~min}$ arc. The responses of one of them are plotted in Figure 14 , in $A$ as a function of number of lines and in $B$ as a function of length of contour. The rising slopes coincide in Figure $14 A$, but not in $B$, i.e., the number of lines, rather than the length, determined the response strength of this neuron. A similar result was obtained in the other neuron.

We tested 9 cells with various spacings of the lines. The stimuli covered the whole receptive field. Figure 15 shows the 2 extremes of the results obtained. The responses of neuron $A$ varied little over the range from 12 to $96 \mathrm{~min}$ arc, while those of neuron $\mathrm{B}$ decreased steeply. The invariance observed in some of the 


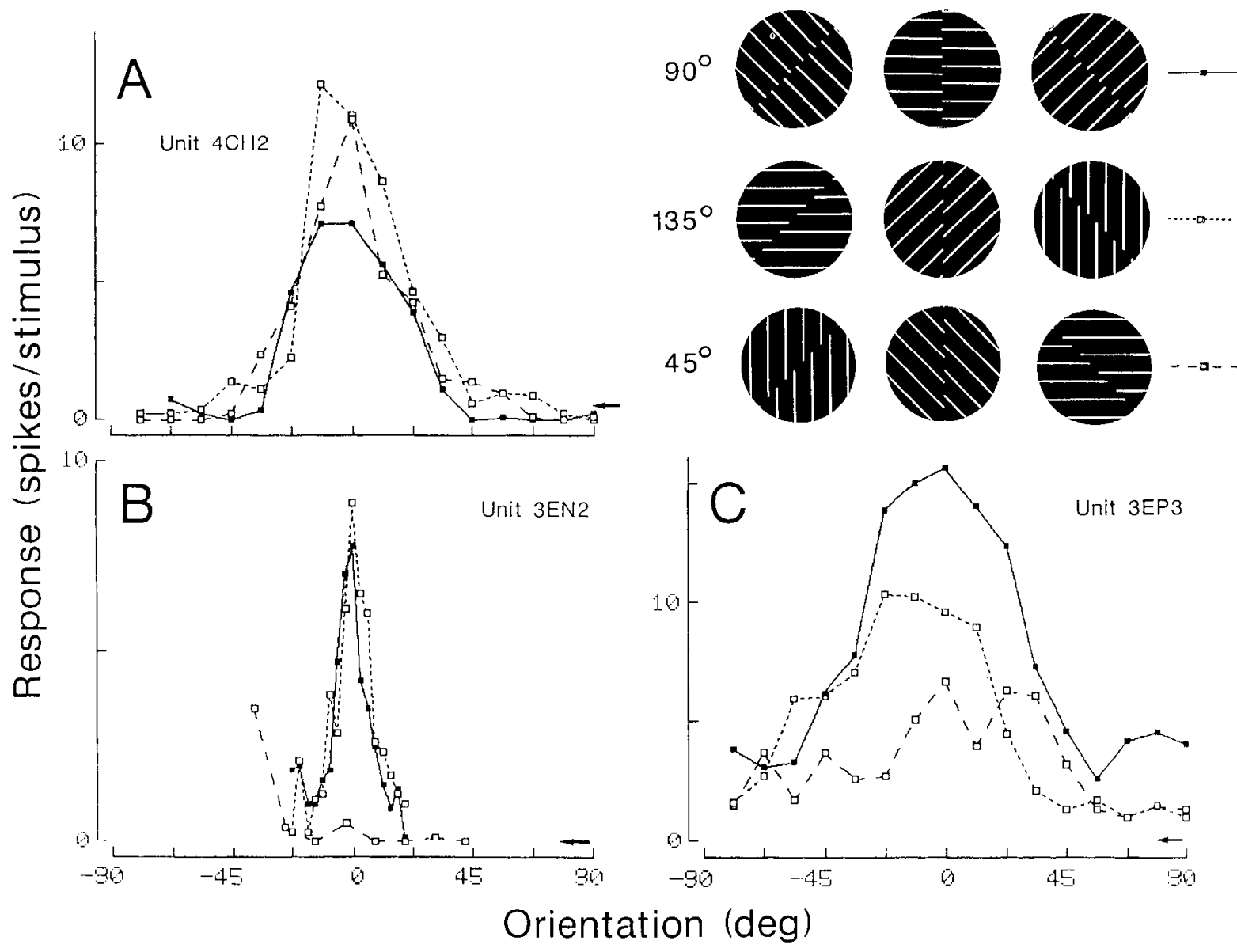

Figure 11. Orientation tuning obtained with anomalous-contours induced by oblique lines in 3 contour cells of V2. Three patterns were tested in which the lines were angled $45^{\circ}, 90^{\circ}$, and $135^{\circ}$ to the contour, respectively. Orientations of the contour, relative to the cell's preferred orientations for bars, are indicated on the abscissae, where positive values mean counter-clockwise rotation (the 3 columns of the stimulus inset correspond to $45^{\circ}, 0^{\circ}$, and $45^{\circ}$ as they appear on the abscissa below). Continuous lines, $90^{\circ}$ condition (our standard stimulus); dashed lines, $45^{\circ}$; dotted lines, $135^{\circ}$. In some cells the peaks coincided $(A)$, i.e., the cell signaled the orientation of the contour irrespective of the angle of the inducing lines; in others, oblique angles caused systematic shifts of the peak $(C)$. The direction of the shift was opposite the direction in which the lines were tilted. Cell $B$ responded with angles of $90^{\circ}$ and $135^{\circ}$ but failed to respond with $45^{\circ}$.

cells is perhaps the most remarkable feature. Note that this observation does not conflict with the conclusion drawn from Figure 14, that the number of lines determined the strength of response at the rising slope of the "length summation curve." The responses may rise proportionately for small numbers, but finally they must reach a maximum ("saturation"). With patterns that cover the whole receptive field, when the density of lines is high, the number of lines within the summation zone may be beyond this limit. With line spacing or $24 \mathrm{~min}$ arc, and the pattern centered in the receptive field, the responses of neuron 3BE1, for example, increased linearly up to about 6 lines and then leveled off (Fig. 14A, filled squares). With large $\left(6^{\circ}\right)$ stimuli, however, the responses were nearly constant for line spacings between 12 and $48 \mathrm{~min}$ arc, and dropped to $56 \%$ at 96 min arc. Apparently, the 48-min-arc pattern provided just the limiting number of line-ends in the summation zone. Since this number was 6 , and the pattern had 2.5 line-ends per degree, the summation zone measured $2^{\circ}-3^{\circ}$, which is consistent with the summation curve for the 48-min-arc pattern plotted in Figure $14 B$ (open squares). The different effects of the density of lines in different neurons might thus reflect the individual levels of saturation and/or weights of the line-ends.
We have seen that most contour cells did not respond to a singlc line of the grating stimulus (Fig. 12). We found only 3 cells that did respond to the end of a line perpendicular to the preferred orientation. An example of such a neuron is represented in Figure 16. The orientation tuning for a single line-end was very broad and, in fact, quite different from the tuning obtained with the whole figure. The additional lines sharpened the tuning in this case. The tuning for a single line-end also differed dramatically from that for a line; the best orientations for the line-end were roughly orthogonal to the optimum line orientation. Line and anomalous contour were nearly equivalent, as in the other contour cells. This neuron may serve as a direct demonstration of the 2 orthogonal inputs postulated in our model (see Discussion); the other contour cells must have these inputs too, but usually several line-ends are needed for a response. It also demonstrates how bizarre responses in V2 can appear if one tries to apply the receptive-field concepts that have been developed for area $\mathrm{V} 1$.

Anomalous-contour responses and conventional length summation. One could ask whether responsiveness to anomalouscontour stimuli is related to conventional length-summation characteristics. Since the number of lines was important, one 
Figure 12. Effect of varying the number of lines on the anomalous-contour responses in 10 cells of V2. Dashed lines indicate the level of spontaneous activity. The density of line-ends on the contour was $2.5 / \mathrm{deg}$ for cell $3 \mathrm{FH} 2,10 / \mathrm{deg}$ for cell $4 \mathrm{BA} 6,5 / \mathrm{deg}$ for all other cells. One line-end usually evoked no response, and maximum responses required many lines. Some neurons had "thresholds" $(a-f)$.

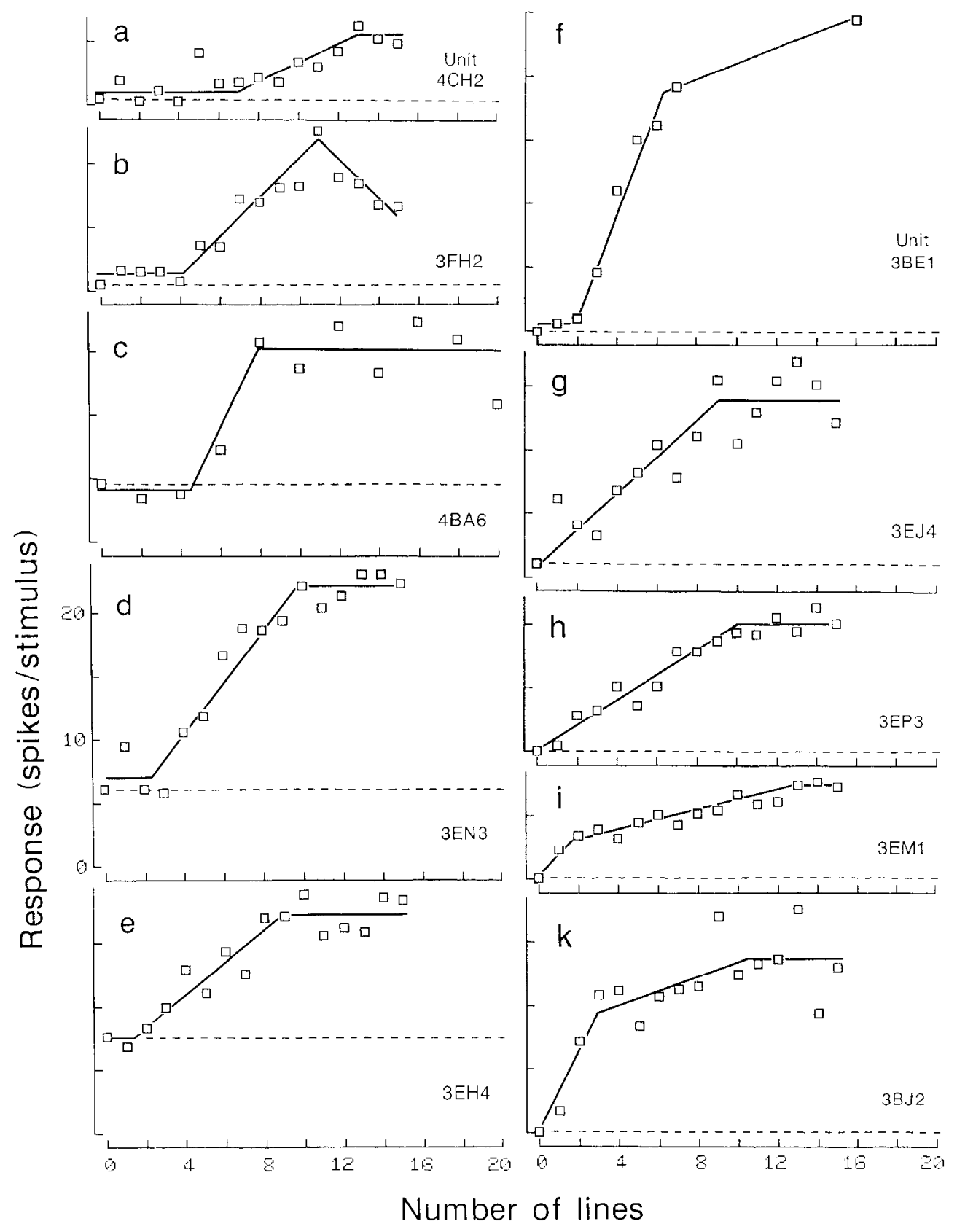

if one attributes some of the differences to response saturation, there remain differences in the rising parts of the curves that are not a matter of scaling in neurons $\mathrm{A}-\mathrm{C}$. Thus, the 2 kinds of stimuli reveal different mechanisms of spatial summation.

\section{Gaps and overlaps}

In the experiments discussed so far, we have used gratings exactly abutting one another for producing anomalous contours. Therefore, the mean luminance taken along lines parallel to the contour was constant over the stimulus. To a classical simple cell that summates along the length dimension these stimuli should thus appear as homogenous fields when the contour is oriented parallel to the receptive field axis. On the other hand, nonlinearities might disturb the balance and make the contour appear. Ends of lines are emphasized by the contrast mechanisms of retina and lateral geniculate nucleus, and length sum- respons. Length summation for bars was extensive neuron $\mathrm{C}$ but moderate or small in the other neurons. One can see also that the 2 curves are dissimilar for each neuron. Even of lines, as in the experiment depicted in Figure 12. The number of lines has been converted into length of contour. Filled squares represent the bar responses, open squares the anomalous-con- 


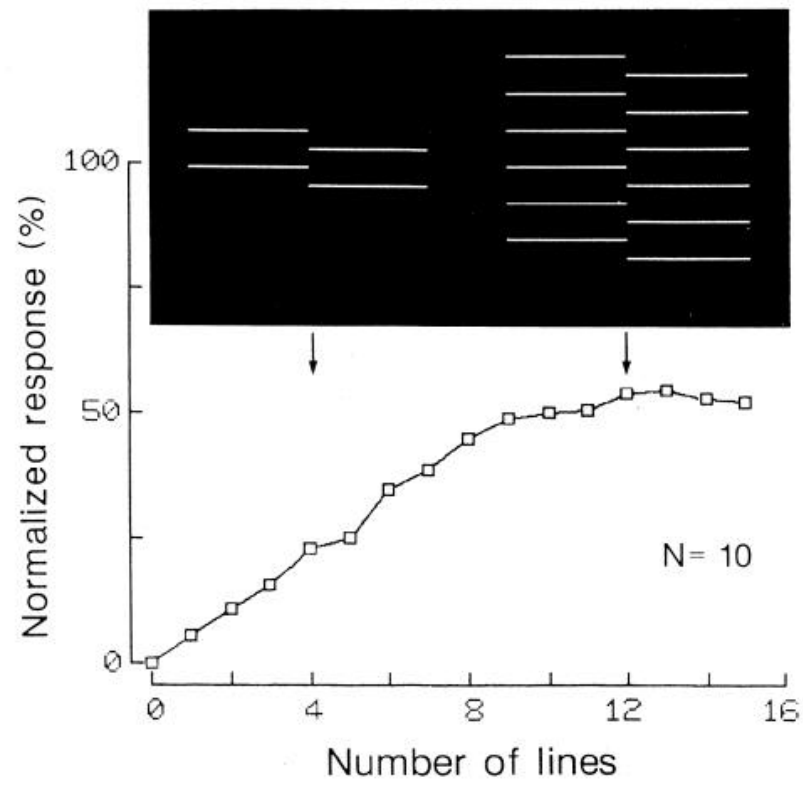

Figure 13. Mean relative strength of the anomalous-contour signals as a function of the number of lines in the stimulus. The response data of Figure 12 have been divided, for each neuron, by the optimum bar or edge response and averaged. The mean shows a nearly linear increase and reaches a maximum at about 12 lines. For a contour with 5 lineends/deg, which was mostly used, this corresponds to a length of $2.4^{\circ}$. The stimuli of half-maximum and maximum mean response are shown in the inset to demonstrate the perceptual difference.

mation in cortical neurons might thus reveal the row of end points as a line. However, our results argue strongly against this possibility since the border between gratings was not detected by cells in V1.

In order to further emphasize the difference between length summation and the mechanism of anomalous-contour responses, we tested 18 neurons, 6 that signaled anomalous contours and 12 that did not, with gratings that were gapping or overlapping to various extents, as shown in Figure 18. The figure shows results from 2 cells of $\mathrm{V} 1$ on the left and 2 contour cells of $\mathrm{V} 2$ on the right. The responses to the grating patterns (open squares) may be compared with the responses to dark and light bars which are plotted with filled squares at the abscissae corresponding to their widths. The cells on the left both preferred

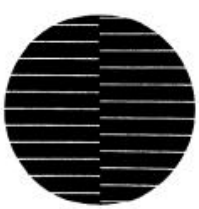

a

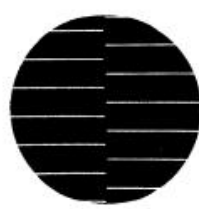

b

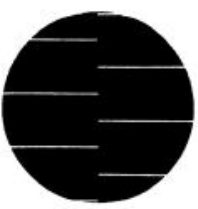

C

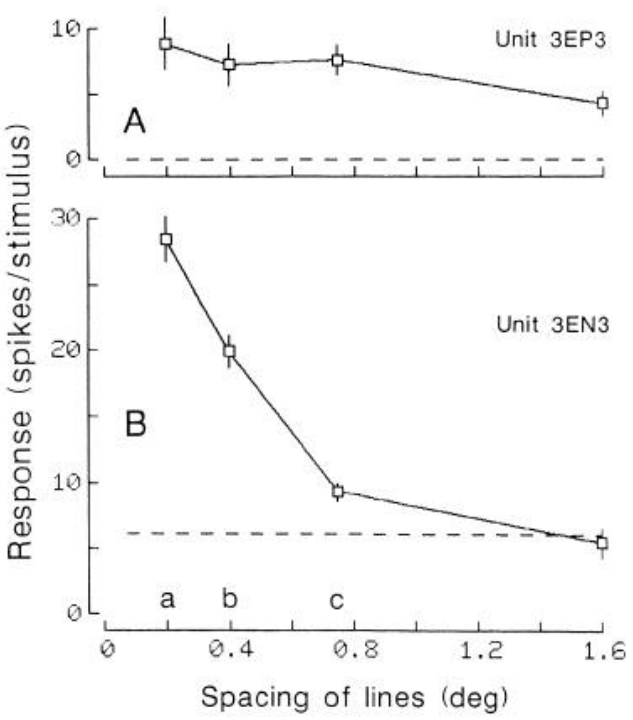

Figure 15. Dependence of the anomalous-contour responses on the density of lines. The examples show the 2 extremes of behavior observed in different neurons. In $B$, responses of the cell dropped to the spontaneous level (dashed line); in $A$, the cell's responses were almost unaffected, when the line spacing was increased from 12 to $96 \mathrm{~min}$ arc. Line width, 1 min arc.

dark bars (left filled square) over light bars (right filled square). It can be seen that they responded to grating patterns with gaps, and less or not at all with overlaps. Cells with light-bar preference responded correspondingly to overlapping gratings. However, the responses fell to zero at the point of exact alignment. Each of the 12 cells in this group showed this drop. By contrast, the neurons that signaled anomalous contours responded to gapping as well as overlapping gratings, including the point of alignment. The responses of neuron $\mathrm{C}$ dropped with increasing overlaps, reaching zero at about $15 \mathrm{~min}$ arc. Indeed,

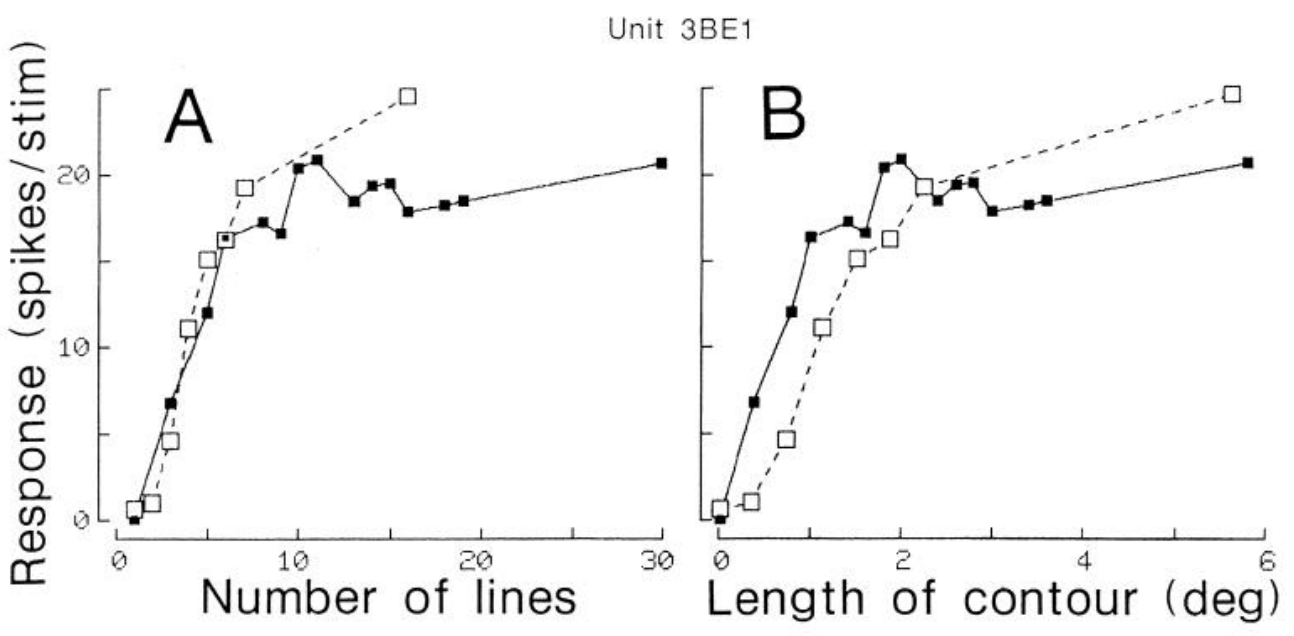

Figure 14. Effect of varying the number of lines for patterns with different line spacings: 24 min arc (filled squares) and $48 \mathrm{~min}$ arc (open squares). In $A$ the responses are plotted versus number of lines; in $B$, versus length of contour. It can be seen that the rising slopes were determined by the number of lines rather than by the length of contour. 


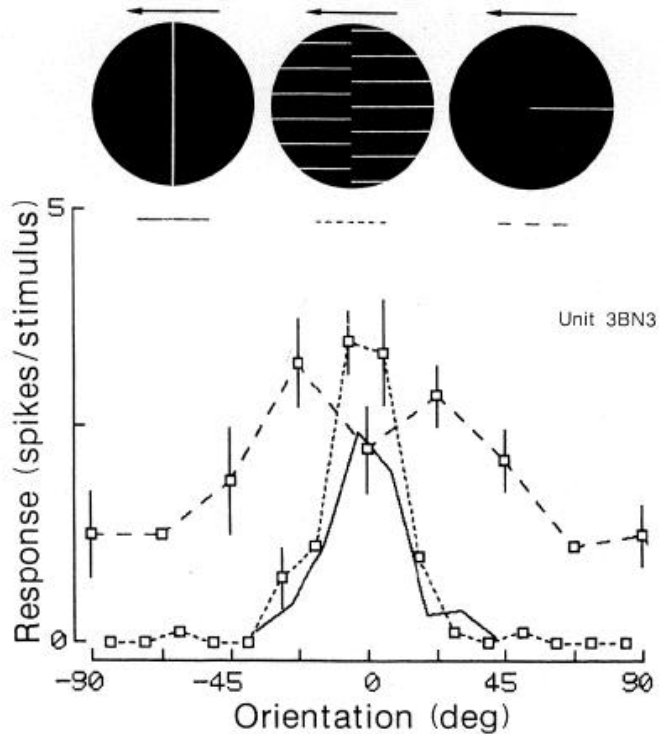

Figure 16. Orientation tuning curves of a contour cell that also responded to the end of a single line orthogonal to its preferred orientation. Continuous curve, light bar; dotted curve with squares, anomalous contour; dashed curve with squares, single line-end. Width of light bar, 2 min arc; width of lines, 1 min arc. Insets show the 3 stimuli at corresponding orientations. Responses to the forward sweeps, as indicated by arrows, are shown. It can be seen that the additional lines sharpened the tuning in this cell. Usually, however, contour cells required several line-ends for a response.

this behavior parallels perception since anomalous contours are vividly perceived at the borders of abutting gratings and gratings separated by a gap (here forming an anomalous dark bar) but less clearly in the case of overlap (see inset in Fig. 18). However, the other cells behaved differently. One showed a peak at zero and small responses on either side; 2 others responded with constant strength over the whole range (one of these is shown in Figure 18D); and in the remaining 2 the responses increased to one or both sides. Another difference between the cells in V1 and the contour cells in V2 was that, in V1, detectable responses to gratings with gaps or overlaps were obtained only with dense gratings, e.g., one line per $12 \mathrm{~min}$ arc, whereas most contour cells responded well with line spacings of 48 , or even 96 , min arc.

One can probably understand these results if one assumes, as will be discussed below, that contour cells have 2 additive inputs, one that is excited by luminance edges or bars and shows length summation (all these neurons did respond to bars or edges) and another that generates the anomalous contour responses. By summation along the length dimension, the edgedetecting mechanism can signal a gap or overlap between the gratings as it signals dim dark or light bars, but it cannot detect the line of discontinuity when the gratings are abutting (Fig. 18, examples at left). The second mechanism probably responds best to the aligned pattern. The weight of the 2 mechanisms varies from cell to cell, as indicated by the relative strengths of edge/bar and anomalous-contour responses. Neuron $\mathrm{C}$ responded weakly to abutting gratings compared with dark bars, and preferred dark over light bars; it thus responded better to gratings with gaps. Neuron D responded better to abutting gratings than to edges and bars, and had not preference for either light or dark bars. In this case, introducing gaps or overlaps may have activated the edge-detecting mechanism which compensated the decline of the anomalous-contour input, resulting in little variation of the total spike counts. With the largest gaps and overlaps, separate responses to the edges of either grating appeared in the dot displays of this cell. Correspondingly, in a neuron that responded to edges better than to abutting gratings, the responses increased in strength with gaps as well as overlaps. We found it hard to make quantitative predictions. The difficulty seems to reside in the fact that even in V1 the responses of most cells change unpredictably if a conventional edge is replaced by a grating edge or a solid bar by a stippled bar: Patterns with equal mean luminance do not produce equal responses; usually the solid pattern is more effective (Peterhans and von der Heydt, 1986; Peterhans et al., 1986). Thus, one cannot predict the strength of the hypothetical edge-detecting
Figure 17. Response strength as a function of length: Comparison between anomalous contours (open squares) and light bars (filled squares) in 4 cells of V2. Abutting gratings of 24 min arc line spacing were used with the number of lines varying between 1 and 15 . The bars were $6(A, B$, and $D)$ or 8 (C) min arc wide. Horizontal dashed lines, spontaneous activity. The anomalous-contour responses could not be predicted from the conventional lengthsummation curves.

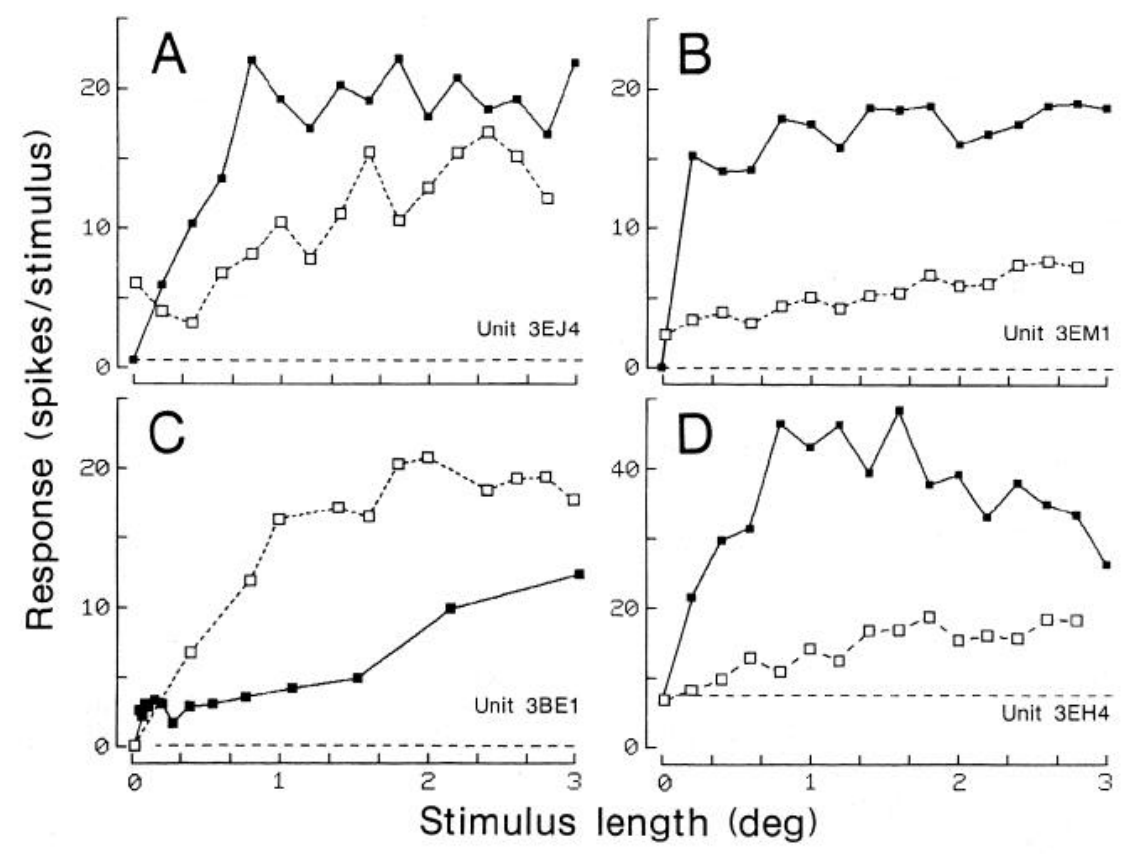



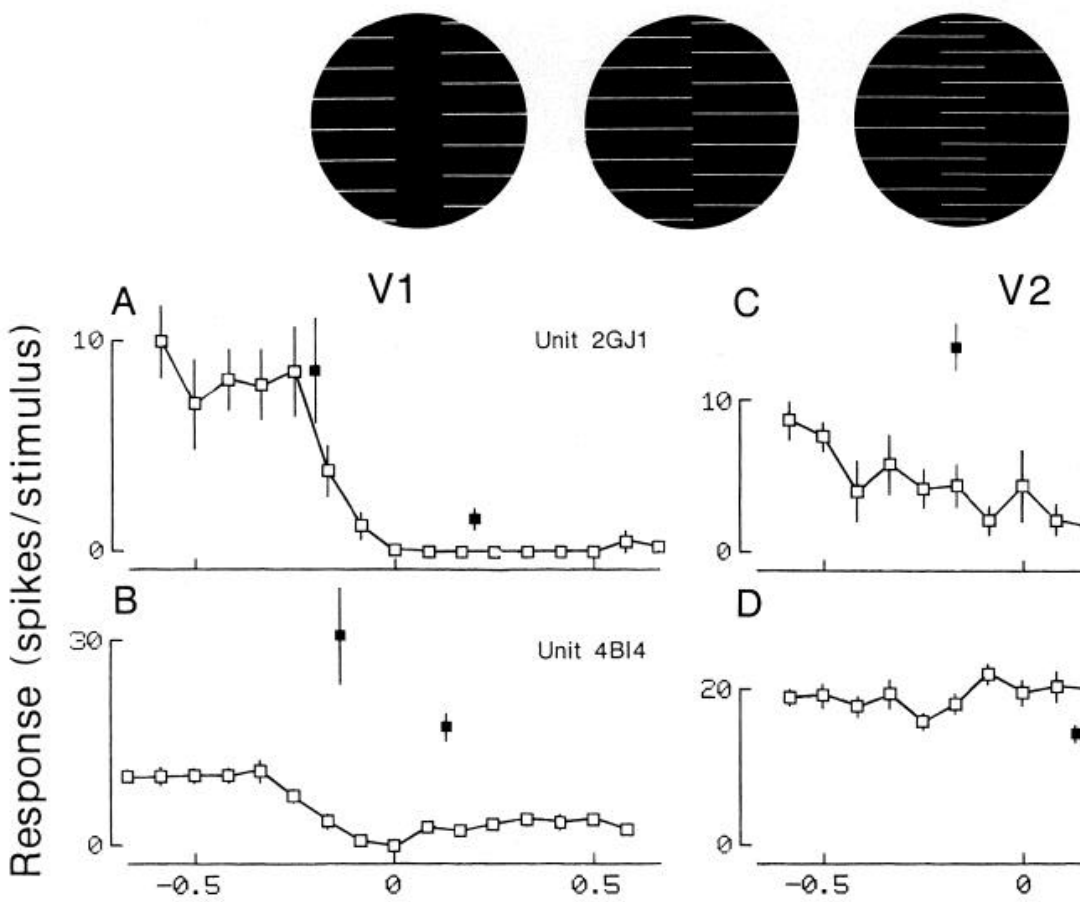

Alignment c
V2 ,

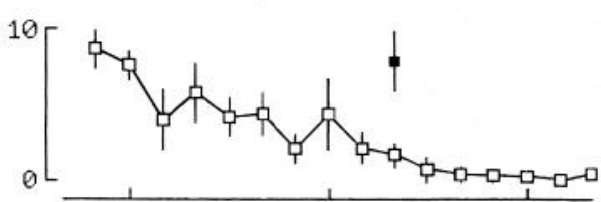

D

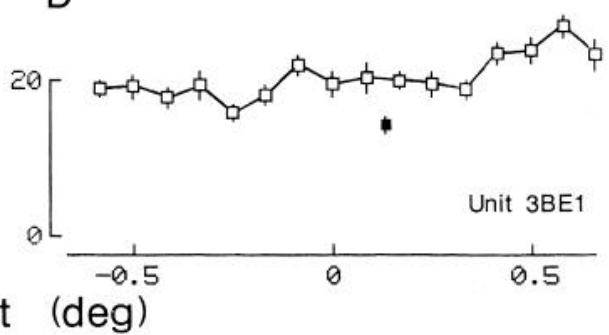

Figure 18. Effect of introducing a gap or overlap between the gratings. Various sizes of gaps and overlaps were tested as indicated on the abscissae, positive: overlaps, negative: gaps. Insets illustrate the abutting condition and the stimuli of maximum gap and overlap $\left(0.67^{\circ}\right)$. Two cells of V1 are represented on the left ( $A$, complex, with darkbar preference, supragranular; $B$, simple, direction selective, infragranular) and 2 contour cells of V2 on the right $(C, D)$. All 4 had very low spontaneous activity. Filled squares represent responses to dark and light bars (unit 3BE1 responded about equally to either bar, but the dark-bar response was not recorded). Cells in Vl often responded to gratings with gaps or overlaps, in accordance with their bar preference, but failed to respond to exactly abutting gratings which activated the contour cells in V2. input to the contour cells in V2 by assuming linear length summation.

\section{Remark on the latency of responses}

Finally, the question of how fast the neural mechanism can determine contours is of interest. Preliminary observations suggest that the anomalous-contour responses have rather short latencies. Figure 19 shows the responses of a cell in V2 to stationary stimuli. The upper panel displays the responses to a light bar turning on and off, and the lower panel responses to a switching anomalous contour: the abutting gratings (Fig. 1C) were alternated with a grating without discontinuity. One can clearly see the pauses before the onsets of activity in either case. The latency was $67 \mathrm{msec}$ for the bar and $77 \mathrm{msec}$ for the anomalous contour. One can also see that the early part of the response was already orientation selective.

\section{Discussion}

The main result of this study is the finding that many neurons in area V2 of the monkey visual cortex signal orientation and position of lines that are perceived as contours but have no counterpart in the stimulus. As an example of such a line we have used the border between 2 abutting gratings, a pattern that differs from other illusory-contour figures in that it has no real line or edge that would indicate the orientation of the contour. The finding indicates a parallel between the responses of these cells and the perception of contour. This result is complemented by the finding that responses related to anomalous contours are practically absent in the primary visual cortex.

Before discussing the details, it may be worthwhile to point out that none of the neurons we have studied was activated exclusively in the presence of anomalous contours. Rather, what we observed in V2 was generalization; contours without gradients appeared to be equivalent to edges or bars. We are limited to such a conclusion by our method, since we used the orien- tation selectivity of the responses to conventional stimuli for interpreting the responses to anomalous-contour stimuli. This equivalence at an early level of cortical processing may account for the illusory aspect of the phenomenon; anomalous contours are confused with edges or lines because both activate the same neurons.

\section{What is represented in the responses?}

We have seen that the orientation of anomalous contours is signaled with the same precision as orientations of edges and bars. There was a range of tuning widths from about $10^{\circ}$ to $70^{\circ}$ in either case, and in general, cells with narrow tuning for conventional stimuli were narrowly tuned also with the anomalous contour, and vice versa. Also, the location and size of the response fields appeared to be similar, although we have not analyzed this aspect quantitatively. All this indicates that configurations that typically form the contours of objects, whether they consist in luminance steps and double-steps (edges and lines) or just in a number of terminations aligned along the contour, are treated similarly in those cells in V2. Our results also show that the responses of neurons in $\mathrm{V} 1$, which are often apostrophized as contour detectors, do not correlate with perception of contour in a general sense. Responses to bars and edges suggested such a correlation, but these are patterns for which contour extraction is trivial. That signals in V1 are not directly related to perception has also been demonstrated in other studies. In checkerboard patterns, for example, cells in V1 did not signal the orientations of the rows that one perceives, but the orientations of the Fourier components (De Valois et al., 1979). Our results confirm the evoked-potential studies in man by Jeffreys (1977), who demonstrated an early, contourspecific component originating from a retinotopically organized area in prestriate cortex. He suggested V2 and V3 as possible sites of the contour-abstraction process.

Our results may not answer the old question of the general 

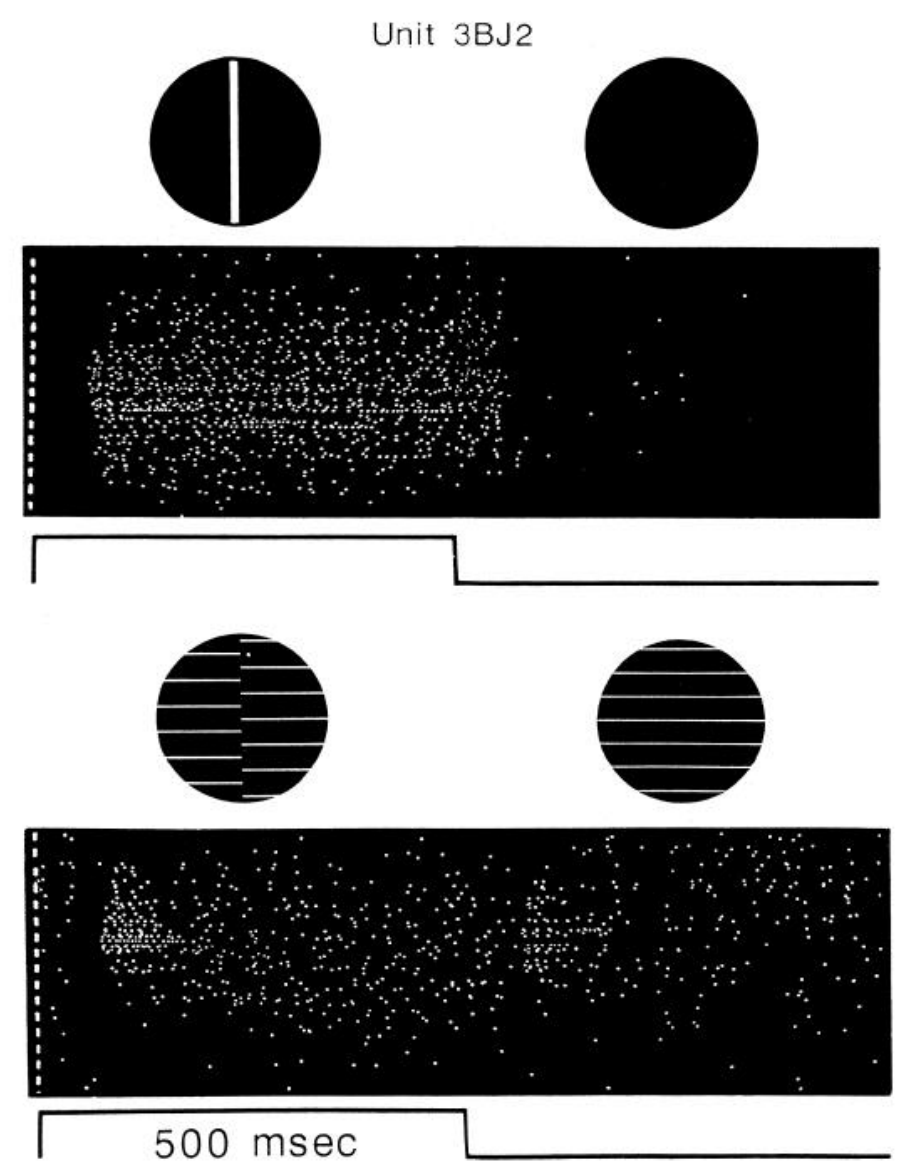

Figure 19. Responses of a contour cell to stimuli switching on and off. Top, Light bar responses; bottom, responses to appearance and disappearance of a line of discontinuity in a grating. Each stimulus was presented at 16 different orientations covering $180^{\circ}$. Note the rather short latencies of about $70-80 \mathrm{msec}$ and the orientation selectivity at the time of response onset. (If the cortical network determined anomalous contours by an iterative process, one would expect the orientation tuning to sharpen gradually after the onset.)

scheme of image coding in the cortex. We still do not know the final goal of the fractionation of image information into orientation bands on what exactly is represented for each orientation. However, the correlation with the perceptual phenomenon of anomalous contours supports the intuitive conviction that determination and encoding of object contours, i.e., segmentation of the image, is a primary issue of visual processing. The fact that such a correlation exists with responses in the secondary visual area V2 suggests that segmentation is attempted at an early stage, presumably before the image context is fully interpreted.

Apparently this fractionation of the image into orientation channels in V2 is totally different from a decomposition into 2-dimensional spatial frequency components which predominates in V1 according to present knowledge (cf. Movshon et al., 1978; Maffei et al., 1979; De Valois et al., 1982; Pollen and Ronner, 1983). Many cells of V2 responded maximally to an anomalous-contour stimulus when the contour had the same orientation as the optimum bar stimulus; however, in the Fourier plane, the bar has all its energy concentrated near the axis perpendicular to its orientation, while the anomalous-contour stimulus has zero energy on this axis (see below). The cells thus signaled an orientation that is not represented at all in the Fourier spectrum. Conversely, often, they did not signal the orientation of the gratings which is heavily represented. Contrast and line density determine the spectral energy and thus would be critical in a frequency-component representation. However, the cells in V2 responded strongly to stimuli consisting of widely spaced thin lines of moderate contrast, and often the density was not critical at all (Fig. 15A).

We have demonstrated anomalous-contour responses mainly with moving stimuli but have also shown examples of responses to stationary patterns. Experiments with stimuli in which a contour was induced by oblique lines and moved obliquely showed that the direction of movement is not the concern of these cells and the orientation of the inducing lines is not the critical parameter either. The cells responded to stimuli consisting of lines perpendicular or oblique to their preferred orientations, but, apart from minor deviations, it was the orientation of the virtual line connecting the line-ends that determined the responses.

When there were deviations from the predicted orientation, these were always in the sense of a rotation away from the inducing lines (Fig. 11), i.e., anomalous contours were signaled with a bias towards the orientation orthogonal to the inducing lines. The perception of these contours is biased in the same way (Pastore, 1971), as Figure $20 B$ demonstrates. This is similar to the Zöllner illusion, which affects the perception of ordinary lines (Fig. 20A). The observation that responses were usually diminished when the inducing lines were tilted also has a per-
Figure 20. A Zöllner illusion with anomalous contours. $A$, An example similar to the classical Zöllner figure; $B$, the long diagonal lines have been replaced by anomalous contours of the type used in this study. The long lines in $A$ are, in fact, parallel, and so are the anomalous contours in $B$. Both appear to be rotated; lines or contours that intercept horizontal lines appear to be rotated toward the vertical, those that intercept vertical lines, toward the horizontal.
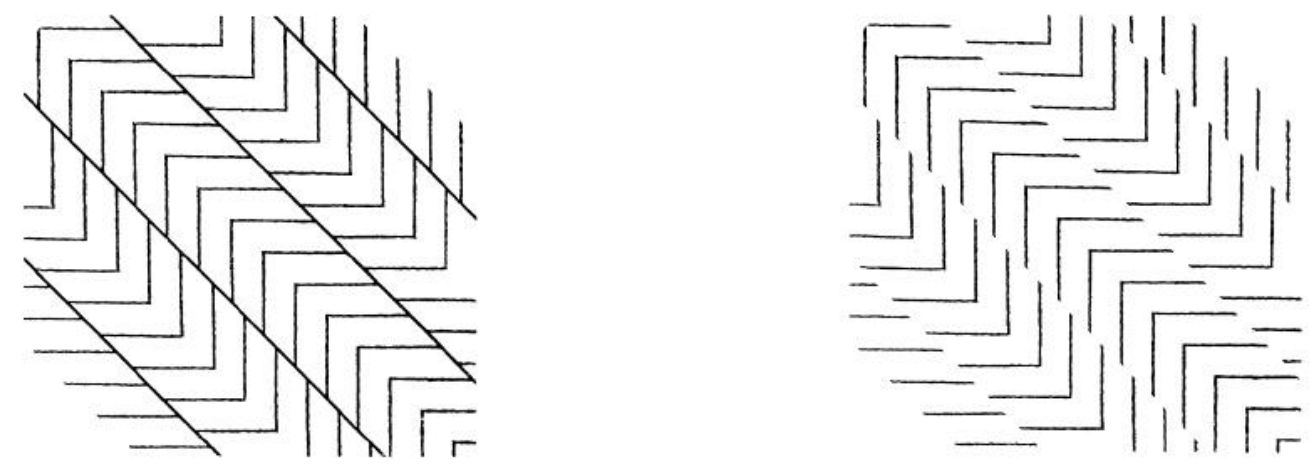
ceptual parallel (Spillmann, 1975; Kennedy, 1978), as demonstrated in Figure 21.

The increase of the neuronal responses with the number of line-ends inducing the anomalous contour (Figs. 12 and 13) is another interesting finding. According to our informal observations, the strength or distinctness of the perceived contours increases similarly (cf. fig. $2 J$ in von der Heydt et al., 1984). We are not aware of direct measurements of this effect, but the brightness of the central patch in the Ehrenstein figure (fig. $21 \mathrm{~A}$, Ehrenstein, 1941) has been measured and shown to increase monotonically with the number of lines (Fuld and O'Donnell, 1984). Even though the anomalous contour is curved in this case, and brightness rather than strength of contour was measured, the underlying mechanism is probably the same.

Our results explain why "illusory contours" produce tilt aftereffects like "real contours" and why the effects transfer (Smith and Over, 1975). Both types of contour are signaled by the same cells. Orientation judgments apparently rely on these signals. Recently, Vogels and Orban (1987) found that training orientation judgments with lines improved performance on lines but not anomalous contours, while training with anomalous contours improved performance on both. They interpret this as showing that there are 2 paths for processing orientation, one activated only by lines and the other by lines and anomalous contours. This might reflect the situation in V2, where some cells sense both types of contour, and others only edges and lines. We do not understand why this experiment shows an asymmetry between lines and anomalous contours, while Smith and Over (1975) found complete equivalence.

There are several observations that do not fit in the analogy with contour perception and are difficult to explain. The effectiveness of the anomalous-contour stimulus relative to an optimally chosen bar or edge varied from cell to cell, and there seemed to be a continuum between unresponsiveness and supernormal responsiveness. Thus, edges and anomalous contours are equivalent in some cells but not in others, and for each orientation there seem to be many different types of cells. Only in 1 of 6 neurons tested did the responses drop when the gratings were made to overlap, but the typical sharp contour (Spinnweblinie) is no longer perceived under this condition. Although, the orientation tuning was generally very similar for bars/edges and anomalous-contour stimuli, we have also seen significant differences that are difficult to interpret. Perhaps we have to consider that anomalous contours are very different from simple edge-type contours, and that there is a large variety of configurations that may define the contours of an interposed object. Because of the known difficulty in reconstructing the contours from these varying substrates, one can hardly expect that neuronal signals at the stage of $\mathrm{V} 2$ are already completely invariant against configuration. As we shall argue, there are probably 2 parallel processes generating the responses of cells of V2, and the above observations of relative independency support this idea.

\section{How are the responses generated?}

\section{Spatial filter theories}

It is sometimes assumed that the "illusory contours" are, in fact, physical edges that can be brought out by suitably filtering the images of the respective figures since this has been demonstrated for certain figures (Ginsburg, 1975; Becker and Knopp, 1978). However, the stimulus we used had equal mean luminance on either side of the anomalous contour. Indeed, the mean
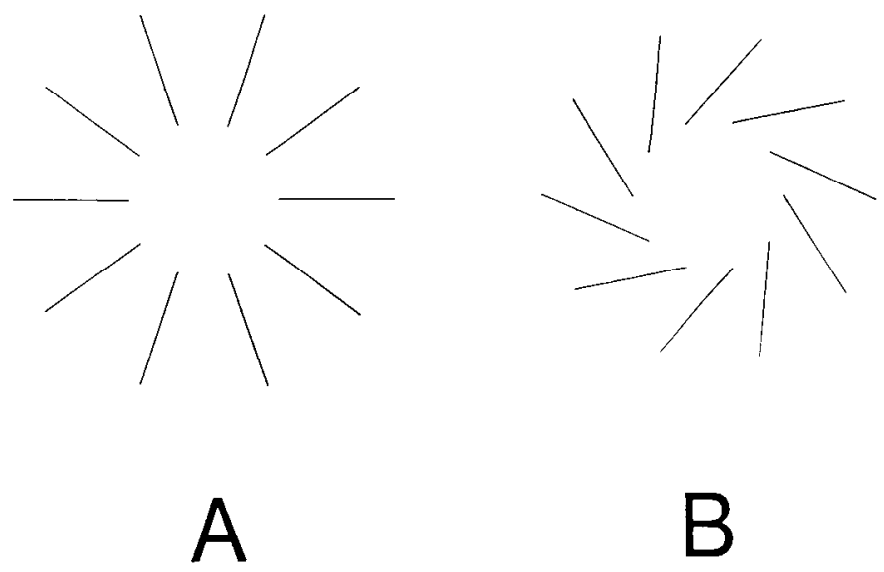

Figure 21. The perception of anomalous contour is strongest when induced by orthogonal lines and becomes weaker when the lines are tilted. (Reproduced, with permission, from Kennedy, 1978.)

taken along lines parallel to the contour was constant all over the figure (disregarding the boundary of the stimulus, which, however, had no influence on neuronal responses). As a consequence, there were no Fourier components of the orientation of the contour, and nothing of that orientation could be brought out by linear filtering. Another figure with this property has been devised by Parks and Pendergrass (1982) to demonstrate the perception of anomalous contours in the absence of corresponding Fourier components. One might think that local spatialfrequency analysis would reveal the contour since looking at small patches of the figure one may see patterns of 1,3 , or 5 lines, etc., in which the mean luminance in fact differs across the contour. Balance is approximated only with large windows. However, the neuronal responses showed just the opposite of what one would expect in this case. When the pattern was masked by windows of various sizes, maximum responses were obtained only with many lines (Fig. 13), and there was no indication of odd numbers being more effective than even numbers.

In fact, our experimental results go farther. The virtual absence of anomalous-contour-related responses in area V1 argues against the possibility that nonlinear transformations in the retinostriate pathway act on the image in such a way that anomalous contours can be revealed by cortical length-summation mechanisms. (We neglect here one exceptional neuron recorded in V1 that did signal the orientation of the anomalous contour.) Neurons that did detect the Fourier components in stimuli with gaps or overlaps between 2 gratings ceased responding at the point of zero overlap, where these components vanish (Fig. 18). These results in V1 are consistent with the demonstrations in cat and monkey that the responses of cells in striate cortex to complex patterns can be predicted to a large extent from the cell's bandpass characteristics and the 2-dimensional Fourier spectrum of the stimulus (cf. De Valois et al., 1979; Maffei et al., 1979). They seem to be at variance, on the other hand, with the recent demonstration in cat that complex, but not simple, cells respond to lines of discontinuity (Redies et al., 1986). We add here that we have often found unresponsiveness even with figures that contain the proper Fourier components; neurons in V1 failed to respond to moving illusory figures where the filter hypothesis predicts responses (Peterhans and von der Heydt, 1989). 
A
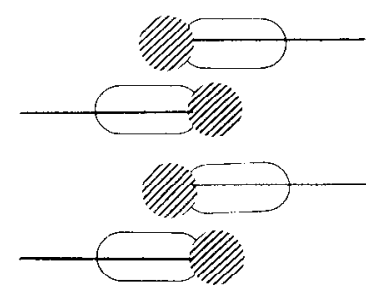
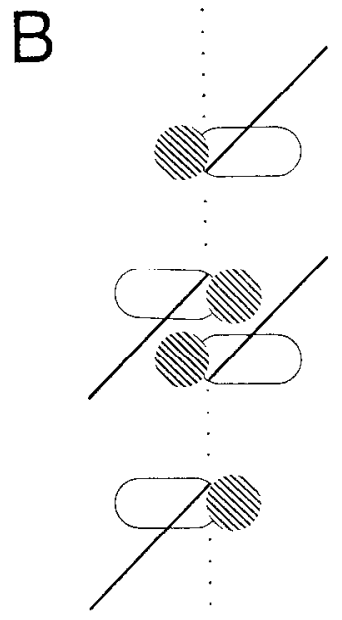

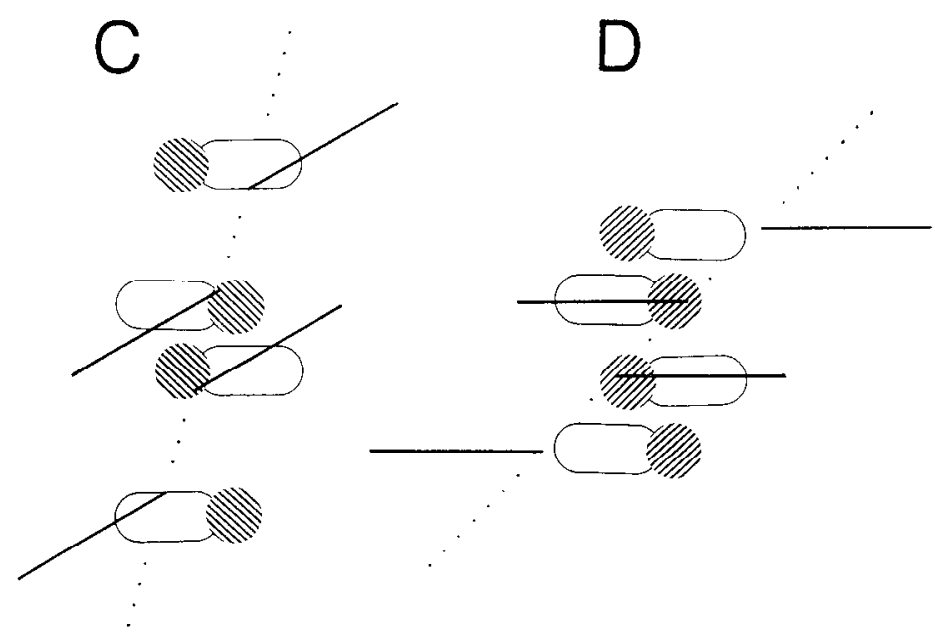

Figure 22. Explanation of the responses to lines of discontinuity, the effect of tilted lines, and the Zöllner illusion. We assume that the contour cells combine edge signals with signals triggered by terminations of edges and lines which occur frequently at occluding contours. The figure shows the case of a neuron signaling "vertical." It receives input from vertically oriented edge-sensitive neurons, which are not illustrated here, and from neurons with end-stopped receptive fields that are lined up along the vertical but have horizontal orientations. Some of these are excited by the abutting gratings as illustrated in $A-D$; only those in the most favorable positions are depicted. For anomalous contours with orthogonal lines, the optimum orientation is vertical $(A)$. With oblique lines, the tuning is biased. In $B-D$, a figure with lines $135^{\circ}$ to the contour is presented at 3 different orientations. When the contour is vertical, the orientation of the lines is far off the preferred orientation of the input fields $(B)$, and when the lines are horizontal, the positions of the ends are not optimal $(D)$; the best orientation is in between $(C)$. This was actually found in the contour cells (Fig. 11C). Thus, our neuron signals "vertical" when the anomalous contour is actually tilting slightly to the right. With lines oblique to the other side $\left(45^{\circ}\right)$, it would signal "vertical" when the anomalous contour would actually be tilting to the left. In other words, the orientation signals of contour cells are biased towards the orientation orthogonal to the inducing lines.

\section{The principle of dual inputs}

This leaves us with the question how the anomalous-contour responses in V2 are generated. The observations that the individual cells showed differential responsiveness to normal and anomalous contours, and that peaks and widths of tuning sometimes differed for the 2 , suggest that these cells receive inputs from 2 sides and that the relative strengths of these inputs vary from neuron to neuron. In an attempt to explain our results by the simplest model, we assume that all the input comes from V1, either directly or via other cells of V2, i.e., we disregard the possibility of centrifugal influences. It is clear, then, that a response to the stimulus of Figure $1 C$ requires excitatory input either from unoriented cells or from cells with orientations perpendicular to that of the target cell since other cells in V1 are not activated by this stimulus. The assumption of unoriented input is unlikely since most of the output of V1 is certainly oriented, and the nonoriented output appears to be specialized for color (Livingstone and Hubel, 1984) and to project to subdivisions of V2 that again contain unoriented cells (Hubel and Livingstone, 1985). Also, the known types of concentric receptive fields cannot form this input because they are also activated by a line grating without discontinuity to which the contour cells of V2 did not respond (Fig. 2). Our demonstration that the orientation of the inducing lines influences the strength of the anomalous contour response (in one neuron a $45^{\circ}$ inclination to one side even abolished the response, Fig. $11 B$ ), as well as the orientation tuning (Fig. $11 C$ ) directly shows that the second input must come from oriented neurons. The most likely candidates are neurons that respond when a properly oriented linecnd or corner is centered in the receptive field but not when a line or edge extends across it. These are a subsample of the cells with strongly end-stopped receptive fields (hypercomplex cells:
Hubel and Wiesel, 1968). We often find such cells in the supragranular layers of V1 (Peterhans and von der Heydt, 1987).

Peterhans et al. (1986) have proposed a model network based on this idea. They assume that, in addition to a conventional input from simple or complex cells, a number of cells with endstopped receptive fields converge on a cell of V2. These fields are lined up in a row with their orientations approximately perpendicular to it. The spatial arrangement of these fields accounts for the sensitivity to the orientation of an anomalous contour and for the summation behavior shown in Figures 12 and 13. Orientation tuning and length summation can thus differ for anomalous contours and conventional stimuli, as found in some cells (see examples in Figs. $5 D$ and 17 , and the distributions in Figs. 7 and 8). The model also implies independent sources of direction selectivity for bars/cdges and anomalous contours, which accounts for the weak correlation of the directionality indices (Fig. 9). The unresponsiveness of the endstopped cells to lines extending across their receptive fields explains why cells signaling the anomalous contour in abutting gratings did not respond to a grating without discontinuity (Fig. $3 B$ ). Finally, the orientation sensitivity of these input cells accounts for the biasing effect of tilted inducing lines (Fig. 11), and the corresponding shift in perceived orientation including the Zöllner illusion (Fig. 20). This is explained in Figure 22. In general, the end-stopped input fields must be oriented roughly orthogonal to the preferred orientation of the target cell in V2, since most cells gave the strongest responses to anomalouscontour figures with orthogonal lines. Occasionally, one can demonstrate this even with a single line-end (Fig. 16). Also theoretically this is the most efficient arrangement because line elements in the background at right angles to the occluding contour are more likely to be intersected by it than line elements with other orientations (von der Heydt and Peterhans, 1987). 
In some cells, however, the end-stopped input fields may be oriented obliquely, rather than orthogonally, in order to suit special stimulus conditions. In these, the best response will be obtained with a contour induced by lines tilted to one side, and no responses with lines tilted to the other side, as was found in neuron B of Figure 11.

End-stopped neurons have been invoked previously to explain the brightness illusion in the Ehrenstein figure (Jung, 1978; Fuld and O'Donnell, 1984; Redies et al., 1984). The concept was different, however. 'The inhibitory end-zones were regarded as part of an antagonistic surround, similar to the surround of concentric receptive ficlds. Cells that arc excited by light bars in the center were assumed to be inhibited by light bars, but not dark bars, in the end-zones, and correspondingly for the opposite center type, resulting in differential inhibition of the 2 types of cell. By pooling the signals of such end-stopped cells irrespective of their orientation, one can construct a light-dark opponent system whose output resembles the perceived brightness distribution (Fuld and O'Donnell, 1984, figure 3). Anomalous contours might be generated from this at a second stage (Frisby and Clatworthy, 1975; Day and Jory, 1980). We think it is unlikely that the system first pools the end-stopped signals, thereby discarding orientation information, and then begins the construction of contours. In our model, end-stopped cells contribute directly to the orthogonal contour signals, and we do not need the (as yet unsupported) assumption that the end-zone inhibition is contrast polarity dependent.

One implication of our model is that neurons representing orthogonal orientations should be connected in V2, and that at least part of these connections should be excitatory. In the cat, Matsubara et al. (1987) have recently shown the intrinsic, patchy connections in area 18 to be most frequent between columns of orthogonal orientations. Assuming that interactions between different orientations serve the sharpening of orientation tuning, they conclude that these connections could only be inhibitory. Assuming that cars have similar contour mechanisms as monkeys, we think that they might well be excitatory. Morphological and immunocytochemical evidence indeed suggests that the patchy projections are excitatory (LeVay, 1988).

The mechanism proposed here accounts also for the results obtained with other contour figures, as presented in the companion paper (Peterhans and von der Heydt, 1989). We defer the further explications and discussion of the alternative theories of illusory contours to that paper.

\section{References}

Becker, M. F., and J. Knopp (1978) Processing of visual illusions in the frequency and spatial domains. Percept. Psychophys. 23: 521526.

Bravo, M., R. Blake, and S. Morrison (1988) Cats see subjective contours. Vision Res. 28: 861-865.

Day, R. H., and M. K. Jory (1980) $\Lambda$ note on a second stage in the formation of illusory contours. Percept. Psychophys. 27: 89-91.

De Valois, K. K., R. L. De Valois, and E. W. Yund (1979) Responses of striate cortex cells to grating and checkerboard patterns. J. Physiol. (Lond.) 291: 483-505.

De Valois, R. L., D. G. Albrecht, and L. G. Thorell (1982) Spatial frequency selectivity of cells in macaque visual cortex. Vision Res. 22: $545-559$.

Ehrenstein, W. (1941) Über Abwandlungen der L. Hermannschen Helligkeitserscheinung. Z. Psychol. 150: 83-91.

Frisby, J. P., and J. L. Clatworthy (1975) Illusory contours: Curious cases of simultaneous brightness contrast? Perception 4: 349-357.
Fuld, K., and K. O'Donnell (1984) Brightness matching and scaling of the Ehrenstein illusion. In Sensory Experience, Adaptation, and Perception. Festschrift for Ivo Kohler. L. Spillmann and B. R. Wooten, eds., pp. 461-469, Lawrence Erlbaum, Hillsdale, NJ.

Ginsburg, A. P. (1975) Is the illusory triangle physical or imaginary? Nature 257: 219-220.

Hubel, D. H., and M. S. Livingstone (1985) Complex-unoriented cells in a subregion of primate area 18 . Nature $315: 325-327$.

IIubel, D. II., and T. N. Wiesel (1965) Receptive ficlds and functional architecture in two non-striate visual areas (18 and 19) of the cat. J. Neurophysiol. 28: 229-289.

Hubel, D. H., and T. N. Wiesel (1968) Receptive fields and functional architecture of monkey striate cortex. J. Physiol. (Lond.) 195: 215 243.

Jeffreys, D. A. (1977) Evoked potential evidence for contour-abstraction in prestriate cortex. J. Physiol. (Lond.) 275: 59P.

Jung, R. (1978) Einführung in die Sehphysiologie. In Physiologie des Menschen, O. H. Gauer, K. Kramer, and R. Jung, eds., pp. 1-140, Urban \& Schwarzenberg, Münich.

Kanizsa, G. (1979) Organization in Vision. Essays on Gestalt Perception, Praeger, New York.

Kennedy, J. M. (1978) Illusory contours and the ends of lines. Perception 7: 605-607.

Koenderink, J. J. (1984) What does the occluding contour tell us about solid shape? Perception 13: 321-330.

Lawson, R. B., and W. L. Gulick (1967) Stereopsis and anomalous contour. Vision Res. 7: 271-297.

LeVay, S. (1988) Patchy intrinsic projections in visual cortex, area 18, of the cat: Morphological and immunocytochemical evidence for an excitatory function. J. Comp. Neurol. 269: 265-274.

Livingstone, M. S., and D. H. Hubel (1984) Anatomy and physiology of a color system in the primate visual cortex. J. Neurosci. 4: 309356.

Maffei, L., C. Morrone, M. Pirchio, and G. Sandini (1979) Responses of visual cortical cells to periodic and non-periodic stimuli. J. Physiol. (Lond.) 296: 27-47.

Marr, D. (1977) Analysis of occluding contour. Proc. R. Soc. London [Biol.] 197: 441-475.

Matsubara, J. A., M. S. Cynader, and N. Y. Swindale (1987) Anatomical properties and physiological correlates of the intrinsic connections in cat area $18 . \mathrm{J}$. Neurosci. $7: 1428-1446$.

Movshon, J. A., I. D. Thompson, and D. J. Tolhurst (1978) Spatial summation in the receptive fields of simple cells in the cat's striate cortex. J. Physiol. (Lond.) 283: 53-77.

Parks, E., and L. Pendergrass (1982) On the filtered-compounds approach to illusory contours. Percept. Psychophys. 32: 491-493.

Pastore, N. (1971) Selective History of Theories of Visual Perception 1650-1950, Oxford U. P., New York.

Peterhans, E., and R. von der Heydt (1986) Cortical neuron responses and perceptual organization. Soc. Neurosci. Abstr. 12: 1499.

Peterhans, E., and R. von der Heydt (1987) The rolc of cnd-stopped receptive fields in contour perception. In New Frontiers in Brain Research: Proceedings of the 15th Göttingen Neurobiology Conference, N. Elsner and O. Creutzfeldt, eds., p. 29, Thieme, Stuttgart.

Peterhans, E., and R. von der Heydt (1989) Mechanisms of contour perception in monkey visual cortex. II. Contours bridging gaps. J. Neurosci. 9: 1749-1763.

Peterhans, E., R. von der Heydt, and G. Baumgartner (1986) Neuronal responses to illusory contour stimuli reveal stages of visual cortical processing. In Visual Neuroscience, J. D. Pettigrew, K. J. Sanderson, and W. R. Levick, eds., pp. 343-351, Cambridge U. P., Cambridge, UK.

Poggio, G. F., R. W. Doty, and W. H. Talbot (1977) Foveal striate cortex of behaving monkey: Single neuron responses to square-wave gratings during fixation of gaze. J. Neurophysiol. 40: 1369-1391.

Pollen, D. A., and S. F. Ronner (1983) Visual cortical neurons as localized spatial frequency filters. IEEE Trans. SMC 13: 907-916.

Redies, C., L. Spillmann, and K. Kunz (1984) Colored neon flanks and line gap enhancement. Vision Res. 24: 1301-1310.

Redies, C., J. M. Crook, and O. D. Creutzfeldt (1986) Neuronal responses to borders with and without luminance gradients in cat visual cortex and dorsal lateral geniculate nucleus. Exp. Brain Res. 61:469481 .

Schumann, F. (1900) Beiträge zur Analyse der Gesichtswahrnehmungen. Erste Abhandlung. Einige Beobachtungen über die Zusammenfassung von Gesichtseindrücken zu Einheiten. Z. Psychol. 23: 1-32. 
Smith, A., and R. Over (1975) Tilt aftereffects with subjective contours. Nature 257: 581-582.

Spillmann, I. (1975) Perceptual modification of the Ehrenstein illusion. In Gestalttheorie der modernen Psychologie, S. Ertel, L. Kemmler, and M. Stadler, eds., pp. 210-218, Steinkopf, Darmstadt. Vogels, R., and G. A. Orban (1987) Illusory contour orientation discrimination. Vision Res. 27: 453-467.

von der Heydt, R., and E. Peterhans (1989) Ehrenstein and Zöllner illusions in a neuronal theory of contour processing. In Seeing Contour and Colour. Proceedings of the 3rd International Symposium of the Northern Eye Institute, Manchester, Aug. 9-13, 1987, C. M. Dickinson and J. J. Kulikowski, eds., Pergamon, London (in press).

von der Heydt, R., E. Peterhans, and G. Baumgartner (1984) Illusory contours and cortical neuron responses. Science 224: 1260-1262.

Wolbarsht, M. L., E. F. MacNichol, Jr., and H. G. Wagner (1960) Glass insulated platinum microelectrode. Science 132: 1309-1310. 